Pacific

Journal of

Mathematics

\title{
CERTAIN SELF-HOMOTOPY EQUIVALENCES ON WEDGE PRODUCTS OF MOORE SPACES
}

Ho Won Choi And Kee Young LeE 


\title{
CERTAIN SELF-HOMOTOPY EQUIVALENCES ON WEDGE PRODUCTS OF MOORE SPACES
}

\author{
Ho Won Choi And KeE Young LEE
}

\begin{abstract}
For a based 1-connected finite $\mathrm{CW}$-complex $X$, let $\mathcal{E}(X)$ denote the group of homotopy classes of self-homotopy equivalences on $X$, and $\mathcal{E}_{\sharp}^{\operatorname{dim}+r}(X)$ the subgroup of $\mathcal{E}(X)$ of homotopy classes of self-homotopy equivalences on $X$ that induce the identity homomorphism on the homotopy groups of $X$ in dimensions $\leq \operatorname{dim} X+r$. For two given Moore spaces $M_{1}=M\left(Z_{q}, n+1\right)$ and $M_{2}=M\left(Z_{p}, n\right)$ with $n \geq 5$, we investigate the subsets of $\left[M_{1}, M_{2}\right]$ and $\left[M_{2}, M_{1}\right]$ consisting of homotopy classes of maps that induce the trivial homomorphism between the homotopy groups of $M_{1}$ and those of $M_{2}$ in dimensions $\leq \operatorname{dim} X+r$. Using the results of this investigation, we completely determine the subgroups $\mathcal{E}_{\sharp}^{\operatorname{dim}+r}\left(M\left(Z_{q}, n+1\right) \vee M\left(Z_{p}, n\right)\right)$, where $p$ and $q$ are positive integers, for $n \geq 5$ and $r=0,1$.
\end{abstract}

\section{Introduction}

If $X$ and $Y$ are based topological spaces, let $[X, Y]$ denote the set of homotopy classes of based maps from $X$ to $Y$, let $\mathcal{E}(X)$ denote the subset of $[X, X]$ that consists of homotopy classes of self-homotopy equivalences of $X$ and let $\mathcal{E}_{\sharp}^{\operatorname{dim}+r}(X)$ denote the set of homotopy classes of self-homotopy equivalences that induce the identity on the homotopy groups of $X$ in $\operatorname{dimensions}$ at most $\operatorname{dim} X+r$. Then, $\mathcal{E}(X)$ is a group with a group operation given by the composition of homotopy classes, and $\mathcal{E}_{\sharp}^{\operatorname{dim}+r}(X)$ is a subgroup of $\mathcal{E}(X)$. The group $\mathcal{E}(X)$ and certain natural subgroups including $\mathcal{E}_{\sharp}^{\operatorname{dim}+r}(X)$ are fundamental objects in homotopy theory and have been studied extensively. For a survey of the known results and applications of $\mathcal{E}(X)$, see [Arkowitz 1990].

When $G$ is an abelian group, we let $M(G, n)$ denote the Moore space, that is, the space with $G$ as a single nonvanishing homology group at $n$-level. Also, in this case, $M(G, n)$ is a simply connected space. We note that if $n \geq 3$, then $M(G, n)$ is characterized by

$$
\widetilde{H}_{i}(M(G, n)) \cong \begin{cases}G & \text { if } i=n \\ 0 & \text { if } i \neq n\end{cases}
$$

This work was supported by a Korea University Grant.

MSC2010: primary 55P10, 55Q05, 55Q20; secondary 55Q40, 55Q52.

Keywords: self-homotopy equivalence, Moore space, homotopy group. 
Let $C(G, n)$ denote the co-Moore space of type $(G, n)$ defined by

$$
\widetilde{H}^{i}(C(G, n)) \cong \begin{cases}G & \text { if } i=n, \\ 0 & \text { if } i \neq n .\end{cases}
$$

If $G$ is a finitely generated abelian group and $G=F \oplus T$, where $F$ is a free abelian group of rank $r$ and $T$ is a finite group, then $M(G, n)=M(F, n) \vee M(T, n)$ and $C(G, n)=M(F, n) \vee M(T, n-1)$ for $n \geq 3$.

Arkowitz and Maruyama [1998] showed that $\mathcal{E}_{\sharp}^{\operatorname{dim}}(M(G, n)) \cong \oplus^{(r+s) s} Z_{2}$ and $\mathcal{E}_{\sharp}^{\operatorname{dim}+1}(M(G, n))=1$ for $n>3$, where $r$ is the rank of $G$ and $s$ is the number of 2-torsion summands in $G$. Moreover, they completely determined $\mathcal{E}_{\sharp}^{\operatorname{dim}}(C(G, n))$ for $n \geq 3$ by means of $2 \times 2$ matrices, where $G$ is a finitely generated abelian group.

Jeong [2010] computed the groups $\mathcal{E}_{\sharp}^{\operatorname{dim}}(Y)$ for $Y=M\left(Z_{p}, n+1\right) \vee M\left(Z_{p}, n\right)$, $n \geq 5$ as follows:

$$
\mathcal{E}_{\sharp}^{\operatorname{dim}}(Y) \cong \begin{cases}Z_{p} & \text { if } p \text { is odd, } \\ Z_{2} \oplus Z_{2} & \text { if } p \equiv 2(\bmod 4), \\ Z_{2} \oplus Z_{2} \oplus Z_{2} & \text { if } p \equiv 0(\bmod 4) .\end{cases}
$$

In this paper we study the self-homotopy equivalences on the wedge product $X=M\left(Z_{q}, n+1\right) \vee M\left(Z_{p}, n\right)$ for $n \geq 5$, where $p$ and $q$ are positive integers. For two given Moore spaces $M_{1}=M\left(Z_{q}, n+1\right)$ and $M_{2}=M\left(Z_{p}, n\right)$, we compute $\left[M_{1}, M_{2}\right]$ and $\left[M_{2}, M_{1}\right]$ and find their generators. Moreover, we investigate the subset of $\left[M_{1}, M_{2}\right]$ or $\left[M_{2}, M_{1}\right]$ that consists of elements whose induced homomorphisms are trivial between the homotopy groups of $M_{1}$ and those of $M_{2}$ in dimensions at most $\operatorname{dim} X+r$ with $r=0,1$. Using these results, we completely determine the groups $\mathcal{E}_{\sharp}^{\operatorname{dim}+r}(X)$ for $r=0,1$. As a result, we obtain Table 1 and the following:

$$
\mathcal{E}_{\sharp}^{\operatorname{dim}+1}(X) \cong \begin{cases}1 & \text { if } q \text { is odd or } p \text { is odd }(d=1), \\ Z_{d} & \text { if } q \text { is odd or } p \text { is odd }(d \neq 1), \\ Z_{d / 2} \oplus Z_{2} & \text { if } p \equiv 0(\bmod 4) \text { and }(p, 24)=4 \text { or } 12(d \neq 1), \\ Z_{d / 2} & \text { if } p \equiv 0(\bmod 4) \text { and }(p, 24)=8 \text { or } 24(d \neq 1), \\ Z_{d / 2} & \text { if } q \equiv 2, p \equiv 2(\bmod 4), \\ Z_{d / 2} \oplus Z_{2} & \text { if } q \equiv 0, p \equiv 2(\bmod 4),\end{cases}
$$

where $d$ is the greatest common divisor of $p$ and $q$.

The space $X$ is neither a Moore space nor a co-Moore space but is characterized by finite homology groups and cohomology groups. That is,

$$
\widetilde{H}_{i}(X) \cong \begin{cases}Z_{p} & \text { if } i=n \\ Z_{q} & \text { if } i=n+1 \\ 0 & \text { otherwise }\end{cases}
$$




\begin{tabular}{lcccc}
\hline & \multicolumn{2}{c}{$q$ is odd } & $q \equiv 2(\bmod 4)$ & $q \equiv 0(\bmod 4)$ \\
& $d=1$ & $d \neq 1$ & & $Z_{2}$ \\
$p$ is odd $(d=1)$ & 1 &. & $Z_{2}$ & $Z_{2} \oplus Z_{d}$ \\
$p$ is odd $(d \neq 1)$ &. & $Z_{d}$ & $Z_{2} \oplus Z_{d}$ & $Z_{2} \oplus Z_{d / 2} \oplus Z_{4}$ \\
$p \equiv 2(\bmod 4)$ & 1 & $Z_{d}$ & $Z_{2} \oplus Z_{d / 2} \oplus Z_{2}$ & $Z_{2} \oplus Z_{d / 2} \oplus Z_{2} \oplus Z_{2}$ \\
$p \equiv 0(\bmod 4)$ & 1 & $Z_{d}$ & $Z_{2} \oplus Z_{d / 2} \oplus Z_{2} \oplus Z_{2}$ & $Z_{2} \oplus{ }^{2}$ \\
\hline
\end{tabular}

Table 1. Isomorphism class of the groups $\mathcal{E}_{\sharp}^{\operatorname{dim}}(X)$.

and

$$
\widetilde{H}^{i}(X, \pi) \cong \begin{cases}\operatorname{Hom}\left(Z_{p}, \pi\right) & \text { if } i=n, \\ \operatorname{Ext}\left(Z_{p}, \pi\right) \oplus \operatorname{Hom}\left(Z_{q}, \pi\right) & \text { if } i=n+1, \\ \operatorname{Ext}\left(Z_{q}, \pi\right) & \text { if } i=n+2, \\ 0 & \text { otherwise. }\end{cases}
$$

From this perspective, $X$ is an interesting space for studying self-homotopy equivalences.

Throughout this paper, all topological spaces are based and have the based homotopy type of a finite l-connected CW-complex. All maps and homotopies will preserve base points. For the spaces $X$ and $Y$, we denote by $[X, Y]$ the set of homotopy classes of maps from $X$ to $Y$. We do not distinguish between the notation of a map $X \rightarrow Y$ and that of its homotopy class in $[X, Y]$. If a group $G$ is generated by a set $\left\{a_{1}, \ldots, a_{n}\right\}$, then we denote the group by $G\left\{a_{1}, \ldots, a_{2}\right\}$ or $G=\left\langle a_{1}, \ldots, a_{n}\right\rangle$.

\section{Preliminaries}

Let $X$ be a space. Then, we denote by $S X$ the suspension of $X$ and by $S^{n} X$ the iterated suspension defined by $S^{n} X=S\left(S^{n-1} X\right)$. Let $f: A \rightarrow B$ be a map and let $C_{f}=B \cup_{f} C A$ be the mapping cone of $f$. Then, we have a Puppe sequence [1958] for $f$,

$$
A \stackrel{f}{\longrightarrow} B \stackrel{i}{\longrightarrow} C_{f} \stackrel{\pi}{\longrightarrow} S A \stackrel{S f}{\longrightarrow} S B \stackrel{S i}{\longrightarrow} S C_{f} \stackrel{S \pi}{\longrightarrow} S^{2} A \stackrel{S^{2} f}{\longrightarrow} S^{2} B \longrightarrow \cdots,
$$

such that the following sequence is exact for any space $X$ :

$$
\cdots \longrightarrow\left[S C_{f}, X\right] \stackrel{S \pi^{*}}{\longrightarrow}[S B, X] \stackrel{S f^{*}}{\longrightarrow}[S A, X] \stackrel{\pi^{*}}{\longrightarrow}\left[C_{f}, X\right] \stackrel{i^{*}}{\longrightarrow}[B, X] \stackrel{f^{*}}{\longrightarrow}[A, X],
$$

where $S^{n} f$ is a suspension map induced by $f$.

If $A$ is $m$-connected and $B$ is $n$-connected, then we have the following exact sequence for any $\mathrm{CW}$-complex $Y$ with dimension at most $m+n$ as a dual sequence 
of the above sequence [Blakers and Massey 1952]:

$$
[Y, A] \stackrel{f_{*}}{\longrightarrow}[Y, B] \stackrel{i_{*}}{\longrightarrow}\left[Y, C_{f}\right] \stackrel{\pi_{*}}{\longrightarrow}[Y, S A] \stackrel{S f_{*}}{\longrightarrow}[Y, S B] \longrightarrow \cdots
$$

Both sequences will be called the exact sequences associated with the cofibration

$$
B \rightarrow C_{f} \rightarrow S A .
$$

Proposition 2.1 [Arkowitz and Maruyama 1998]. If $X$ is $(k-1)$-connected, $Y$ is $(l-1)$-connected, $k, l \geq 2$ and $\operatorname{dim} P \leq k+l-1$, then the projections $X \vee Y \rightarrow X$ and $X \vee Y \rightarrow Y$ induce a bijection

$$
[P, X \vee Y] \rightarrow[P, X] \oplus[P, Y] .
$$

Proposition 2.1 is a consequence of [Spanier 1966, p. 405] since the inclusion $X \vee Y \rightarrow X \times Y$ is a $(k+l-1)$-equivalence.

Next, we consider abelian groups $G_{1}$ and $G_{2}$ and Moore spaces $M_{1}=M\left(G_{1}, n_{1}\right)$ and $M_{2}=M\left(G_{2}, n_{2}\right)$. Let $X=M_{1} \vee M_{2}$. We denote by $i_{j}: M_{j} \rightarrow X$ the inclusion and by $p_{j}: X \rightarrow M_{j}$ the projection, where $j=1,2$. If $f: X \rightarrow X$, then we define $f_{j k}: M_{k} \rightarrow M_{j}$ by $f_{j k}=p_{j} f i_{k}$ for $j, k=1,2$.

If $f: X \rightarrow Y$ is a map, then $f_{\sharp n}: \pi_{n}(X) \rightarrow \pi_{n}(Y)$ denotes the induced homomorphism in dimension $n$.

Proposition 2.2 [Arkowitz and Maruyama 1998]. The function $\theta$ that assigns to each $f \in[X, X]$ the $2 \times 2$ matrix

$$
\theta(f)=\left(\begin{array}{ll}
f_{11} & f_{12} \\
f_{21} & f_{22}
\end{array}\right)
$$

where $f_{j k} \in\left[M_{k}, M_{j}\right]$, is a bijection. In addition:

(1) $\theta(f+g)=\theta(f)+\theta(g)$, so $\theta$ is an isomorphism $[X, X] \rightarrow \bigoplus_{j, k=1,2}\left[M_{k}, M_{j}\right]$.

(2) $\theta(f g)=\theta(f) \theta(g)$, where $f g$ denotes composition in $[X, X]$ and $\theta(f) \theta(g)$ denotes matrix multiplication.

(3) If $\alpha_{r}: \pi_{r}\left(M_{1}\right) \oplus \pi_{r}\left(M_{2}\right) \rightarrow \pi_{r}\left(M_{1} \vee M_{2}\right)$ is the homomorphism induced by the inclusions and $\beta_{r}: \pi_{r}\left(M_{1} \vee M_{2}\right) \rightarrow \pi_{r}\left(M_{1}\right) \oplus \pi_{r}\left(M_{2}\right)$ the homomorphism induced by the projections respectively, then

$$
\beta_{r} f_{\sharp r} \alpha_{r}(x, y)=\left(f_{11 \sharp r}(x)+f_{12 \sharp r}(y), f_{21 \sharp r}(x)+f_{22 \sharp r}(y)\right)
$$

for $x \in \pi_{r}\left(M_{1}\right)$ and $y \in \pi_{r}\left(M_{2}\right)$.

Proposition 2.3 [Araki and Toda 1965]. (1) $\pi_{n}\left(M\left(Z_{q}, n\right)\right) \cong Z_{q}$ for all $q$.

(2) $\pi_{n+1}\left(M\left(Z_{q}, n\right)\right) \cong \begin{cases}0 & \text { if } q \text { is odd }, \\ Z_{2} & \text { if } q \text { is even. }\end{cases}$ 
(3) $\pi_{n+2}\left(M\left(Z_{q}, n\right)\right) \cong \begin{cases}0 & \text { if } q \text { is odd, } \\ Z_{4} & \text { if } q \equiv 2(\bmod 4), \\ Z_{2} \oplus Z_{2} & \text { if } q \equiv 0(\bmod 4) \text {. }\end{cases}$

(4) $\pi_{n+3}\left(M\left(Z_{q}, n\right)\right) \cong \begin{cases}Z_{(q, 24)} & \text { if } q \text { is odd }, \\ Z_{(q, 24)} \oplus Z_{2} & \text { if } q \text { is even. }\end{cases}$

The generators of $\left[S^{n+i}, S^{n}\right]$ can be summarized thus [Toda 1962]:

$\begin{array}{ccccccc} & i<0 & i=0 & i=1 & i=2 & i=3 & i=4,5 \\ {\left[S^{n+i}, S^{n}\right]} & 0 & Z & Z_{2} & Z_{2} & Z_{24} & 0 \\ \text { Generator } & & \iota & \eta & \eta^{2} & v & 0\end{array}$

Proposition 2.4 [Araki and Toda 1965].

(1) $\left[M\left(Z_{q}, n\right), S^{n}\right] \cong \begin{cases}0 & \text { if } q \text { is odd }, \\ Z_{2} & \text { if } q \text { is even. }\end{cases}$

(2) $\left[M\left(Z_{q}, n+1\right), S^{n}\right] \cong \begin{cases}0 & \text { if } q \text { is odd, } \\ Z_{4} & \text { if } q \equiv 2(\bmod 4) \text {, } \\ Z_{2} \oplus Z_{2} & \text { if } q \equiv 0(\bmod 4) \text {. }\end{cases}$

Proposition 2.5 [Arkowitz and Maruyama 1998]. For the Moore space $X=$ $M(G, n)$ :

(1) $\mathcal{E}_{\sharp}^{\operatorname{dim}}(X) \cong \oplus^{(r+s) s} Z_{2}$, where $r$ is the rank of $G$ and $s$ is the number of 2torsion summands in $G$.

(2) $\mathcal{E}_{\sharp}^{\operatorname{dim}+1}(X) \cong 1$ if $n>3$.

Proposition 2.6 (universal coefficient theorem for homotopy groups with coefficients [Hilton 1965]). There is an exact sequence

$$
0 \rightarrow \operatorname{Ext}\left(G, \pi_{n+1}(X)\right) \rightarrow \pi_{n}(G ; X) \rightarrow \operatorname{Hom}\left(G, \pi_{n}(X)\right) \rightarrow 0,
$$

where $\pi_{n}(G ; X)$, the $n$-th homotopy group of $X$ with coefficients in $G$, is given by $\pi_{n}(G ; X)=[M(G, n), X]$, where $M(G, n)$ is a Moore space.

\section{Generators of the sets of homotopy classes on Moore spaces}

In this section, we find generators of homotopy groups of Moore spaces and the sets of homotopy classes between two Moore spaces. Let

$$
M_{1}=M\left(Z_{q}, n+1\right)=S^{n+1} \cup_{q} e^{n+2} \quad \text { and } \quad M_{2}=M\left(Z_{p}, n\right)=S^{n} \cup_{p} e^{n+1},
$$

with $p, q \geq 1$. Then, there are two mapping cone sequences

$$
S^{n+1} \stackrel{q_{\iota_{1}}}{\longrightarrow} S^{n+1} \stackrel{i_{1}}{\longrightarrow} S^{n+1} \cup_{q} e^{n+2} \stackrel{\pi_{1}}{\longrightarrow} S^{n+2} \stackrel{q_{\iota_{1}}}{\longrightarrow} S^{n+2}
$$


and

$$
S^{n} \stackrel{p_{t_{2}}}{\longrightarrow} S^{n} \stackrel{i_{2}}{\longrightarrow} S^{n} \cup_{p} e^{n+1} \stackrel{\pi_{2}}{\longrightarrow} S^{n+1} \stackrel{p_{t_{2}}}{\longrightarrow} S^{n+1},
$$

where $p_{\iota_{2}}$ and $q_{\iota_{1}}$ are maps with degree $p$ and $q$ respectively.

Remark 3.1. We find generators of $\pi_{m}\left(M\left(Z_{r}, n\right)\right)$, for $n \leq m \leq n+2$.

Recall that $\pi_{n}\left(M\left(Z_{r}, n\right)\right) \cong Z_{r}$. From the mapping cone sequence

$$
S^{n} \stackrel{r_{\iota}}{\longrightarrow} S^{n} \stackrel{i}{\longrightarrow} M\left(Z_{r}, n\right) \stackrel{\pi}{\longrightarrow} S^{n+1} \stackrel{r_{\iota}}{\longrightarrow} S^{n+1},
$$

we obtain the long exact sequence

$$
\pi_{n}\left(S^{n}\right) \stackrel{r_{\sharp \sharp}}{\longrightarrow} \pi_{n}\left(S^{n}\right) \stackrel{i_{\sharp}}{\longrightarrow} \pi_{n}\left(M\left(Z_{r}, n\right)\right) \stackrel{\pi_{\sharp}}{\longrightarrow} \pi_{n}\left(S^{n+1}\right) \stackrel{r_{\iota \sharp}}{\longrightarrow} \pi_{n}\left(S^{n+1}\right) .
$$

By the results in [Toda 1962], we have the sequence

$$
Z\{\iota\} \stackrel{r_{\iota \sharp}}{\longrightarrow} Z\{\iota\} \stackrel{i_{\sharp}}{\longrightarrow} \pi_{n}\left(M\left(Z_{r}, n\right)\right) \longrightarrow 0,
$$

so $i_{\sharp}$ is surjective. Thus, $\pi_{n}\left(M\left(Z_{r}, n\right)\right) \cong Z\{\iota\} / \operatorname{Im}\left(r_{\iota \sharp}\right)$. Let $i_{\sharp}(\iota)=i$. Then, we can take $i$ as a generator of $\pi_{n}\left(M\left(Z_{r}, n\right)\right)$.

Next, we find a generator of $\pi_{n+1}\left(M\left(Z_{r}, n\right)\right)$. There are two cases according to the parity of the positive integer $r$. If $r$ is odd, then $\pi_{n+1}\left(M\left(Z_{r}, n\right)\right)$ is trivial. If $r$ is even, then we can take $i_{\sharp}(\eta)$ as a generator of $\pi_{n+1}\left(M\left(Z_{r}, n\right)\right)$, where $\eta$ is the generator of $\pi_{n+1}\left(S^{n}\right)$.

Finally, we find a generator of $\pi_{n+2}\left(M\left(Z_{r}, n\right)\right)$. Consider the exact sequence

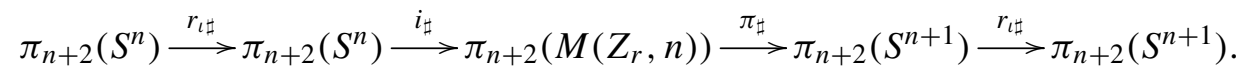

Then by the results in [Toda 1962], we have the exact sequence

$$
Z_{2}\left\{\eta^{2}\right\} \stackrel{r_{\sharp \sharp}}{\longrightarrow} Z_{2}\left\{\eta^{2}\right\} \stackrel{i_{\sharp}}{\longrightarrow} \pi_{n+2}\left(M\left(Z_{r}, n\right)\right) \stackrel{\pi_{\sharp}}{\longrightarrow} Z_{2}\{\eta\} \stackrel{q_{\sharp \sharp}}{\longrightarrow} Z_{2}\{\eta\} .
$$

Since $r$ is an even number, we obtain the exact sequence

$$
0 \longrightarrow Z_{2}\left\{\eta^{2}\right\} \stackrel{i_{\sharp}}{\longrightarrow} \pi_{n+2}\left(M\left(Z_{r}, n\right)\right) \stackrel{\pi_{\sharp}}{\longrightarrow} Z_{2}\{\eta\} \longrightarrow 0 .
$$

If $r \equiv 2(\bmod 4)$, then $\pi_{n+2}\left(M\left(Z_{r}, n\right)\right) \cong Z_{4}\{\bar{\eta}\}$ such that $i_{\sharp}\left(\eta^{2}\right)=2 \bar{\eta}$ and $\pi_{\sharp}(\bar{\eta})=\eta$. On the other hand, if $r \equiv 0(\bmod 4)$, then $\pi_{n+2}\left(M\left(Z_{r}, n\right)\right) \cong Z_{2} \oplus Z_{2}\left\{\eta_{1}, \eta_{2}\right\}$ such that $i_{\sharp}\left(\eta^{2}\right)=\eta_{1}$ and $\pi_{\sharp}\left(\eta_{2}\right)=\eta$.

By Remark 3.1, it follows that

$$
\begin{array}{lrl}
\pi_{n+1}\left(M_{1}\right) \cong Z_{q}\left\{i_{1}\right\}, & \pi_{n}\left(M_{2}\right) \cong Z_{p}\left\{i_{2}\right\}, \\
\pi_{n+2}\left(M_{1}\right) \cong Z_{2}\left\{i_{1 \sharp}(\eta)\right\}, & \pi_{n+1}\left(M_{2}\right) \cong Z_{2}\left\{i_{2 \sharp}(\eta)\right\} .
\end{array}
$$

Moreover, $\pi_{n+2}\left(M_{2}\right) \cong Z_{4}\{\bar{\eta}\}$ or $\pi_{n+2}\left(M_{2}\right) \cong Z_{2} \oplus Z_{2}\left\{\eta_{1}, \eta_{2}\right\}$. 
Lemma 3.2. Let $p$ and $q$ be positive integers and $(p, q)$ be the greatest common divisor of $p$ and $q$. Consequently, if $(p, q)=d \neq 1$, then $\left[M_{2}, M_{1}\right] \cong Z_{d}\left\{\pi_{2}^{*}\left(i_{1}\right)\right\}$ and if $(p, q)=1$, then $\left[M_{2}, M_{1}\right] \cong 0$.

Proof. Consider the mapping cone sequence of $M_{2}$,

$$
S^{n} \stackrel{p_{\iota_{2}}}{\longrightarrow} S^{n} \stackrel{i_{2}}{\longrightarrow} S^{n} \cup_{p} e^{n+1} \stackrel{\pi_{2}}{\longrightarrow} S^{n+1} \stackrel{p_{\iota_{2}}}{\longrightarrow} S^{n+1} .
$$

This sequence induces the following exact sequence:

$$
\pi_{n+1}\left(M_{1}\right) \stackrel{p_{t_{2}} *}{\longrightarrow} \pi_{n+1}\left(M_{1}\right) \stackrel{\pi_{2} *}{\longrightarrow}\left[M_{2}, M_{1}\right] \stackrel{i_{2} *}{\longrightarrow} \pi_{n}\left(M_{1}\right) \stackrel{p_{t_{2}} *}{\longrightarrow} \pi_{n}\left(M_{1}\right) .
$$

Since $\pi_{n+1}\left(M_{1}\right) \cong Z_{q}\left\{i_{1}\right\}$ and $\pi_{n}\left(M_{1}\right) \cong 0$, the exact sequence above becomes

$$
Z_{q}\left\{i_{1}\right\} \stackrel{p_{\iota_{2}}^{*}}{\longrightarrow} Z_{q}\left\{i_{1}\right\} \stackrel{\pi_{2}^{*}}{\longrightarrow}\left[M_{2}, M_{1}\right] \longrightarrow 0 .
$$

If $(p, q)=1$, the first $p_{\iota_{2}}^{*}$ is an isomorphism, so $\left[M_{2}, M_{1}\right] \cong 0$. Let $(p, q)=d \neq 1$. Then, since $\pi_{2}^{*}$ is surjective and $p_{\iota_{2}}^{*}\left(i_{1}\right)=p i_{1}$, we have

$$
\left[M_{2}, M_{1}\right]=\operatorname{im} \pi_{2}^{*} \cong Z_{q}\left\{i_{1}\right\} / \operatorname{im} p_{\iota_{2}}^{*} \cong Z_{d}\left\{\pi_{2}^{*}\left(i_{1}\right)\right\} .
$$

Lemma 3.3. If $p$ or $q$ is odd, then $\left[M_{1}, M_{2}\right] \cong 0$.

Proof. Consider the mapping cone sequence of $M_{1}$,

$$
S^{n+1} \stackrel{q_{\iota_{1}}}{\longrightarrow} S^{n+1} \stackrel{i_{1}}{\longrightarrow} S^{n+1} \cup_{q} e^{n+2} \stackrel{\pi_{1}}{\longrightarrow} S^{n+2} \stackrel{q_{\iota_{1}}}{\longrightarrow} S^{n+2} .
$$

Then, we have the exact sequence

$$
\pi_{n+2}\left(M_{2}\right) \stackrel{q_{\iota_{1}}^{*}}{\longrightarrow} \pi_{n+2}\left(M_{2}\right) \stackrel{\pi_{1}^{*}}{\longrightarrow}\left[M_{1}, M_{2}\right] \stackrel{i_{1}^{*}}{\longrightarrow} \pi_{n+1}\left(M_{2}\right) \stackrel{q_{\iota_{1}}^{*}}{\longrightarrow} \pi_{n+1}\left(M_{2}\right) .
$$

Let $p \equiv 2(\bmod 4)$ and let $q$ be odd. Then, since $\pi_{n+1}\left(M_{2}\right) \cong Z_{2}$ and $\pi_{n+2}\left(M_{2}\right) \cong Z_{4}$, we have the sequence

$$
Z_{4} \stackrel{q_{\iota_{1}}^{*}}{\longrightarrow} Z_{4} \stackrel{\pi_{1}^{*}}{\longrightarrow}\left[M_{1}, M_{2}\right] \stackrel{i_{1}^{*}}{\longrightarrow} Z_{2} \stackrel{q_{\iota_{1}}^{*}}{\longrightarrow} Z_{2} .
$$

Furthermore, since $(q, 4)=1$ and $(q, 2)=1$, each $q_{\iota_{1}}^{*}$ is an isomorphism. Thus we have the exact sequence

$$
0 \rightarrow\left[M_{1}, M_{2}\right] \rightarrow 0
$$

Therefore, $\left[M_{1}, M_{2}\right] \cong 0$.

In the case where $p \equiv 0(\bmod 4)$ and $q$ is odd, we can give a similar proof.

Next, let $p$ be odd. Since $\pi_{n+1}\left(M_{2}\right)$ and $\pi_{n+2}\left(M_{2}\right)$ are trivial groups, so is $\left[M_{1}, M_{2}\right]$ by exactness. 
Let $p$ and $q$ be even. From the exact sequences associated with the cofibrations $S^{n+1} \rightarrow M_{1} \rightarrow S^{n+2}$ and $S^{n} \rightarrow M_{2} \rightarrow S^{n+1}$, we have the following commutative diagram with exact rows and columns:

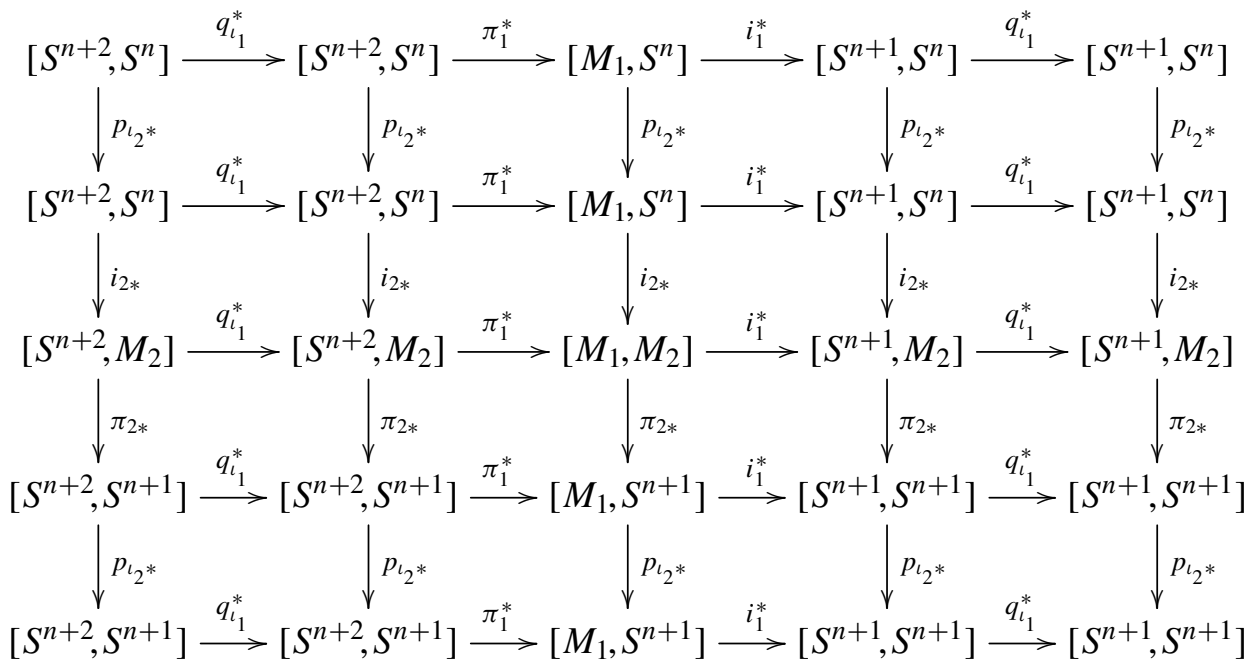

Lemma 3.4. Let $(p, q) \neq 1$. Then, if either $p \equiv 0(\bmod 4)$ and $q \equiv 2(\bmod 4)$ or $p \equiv 2(\bmod 4)$ and $q \equiv 0(\bmod 4)$, we have $\left[M_{1}, M_{2}\right] \cong Z_{4} \oplus Z_{2}$.

Proof. Suppose that $p \equiv 0(\bmod 4)$ and $q \equiv 2(\bmod 4)$. With the results in [Araki and Toda 1965], we obtain the following diagram from the above diagram:

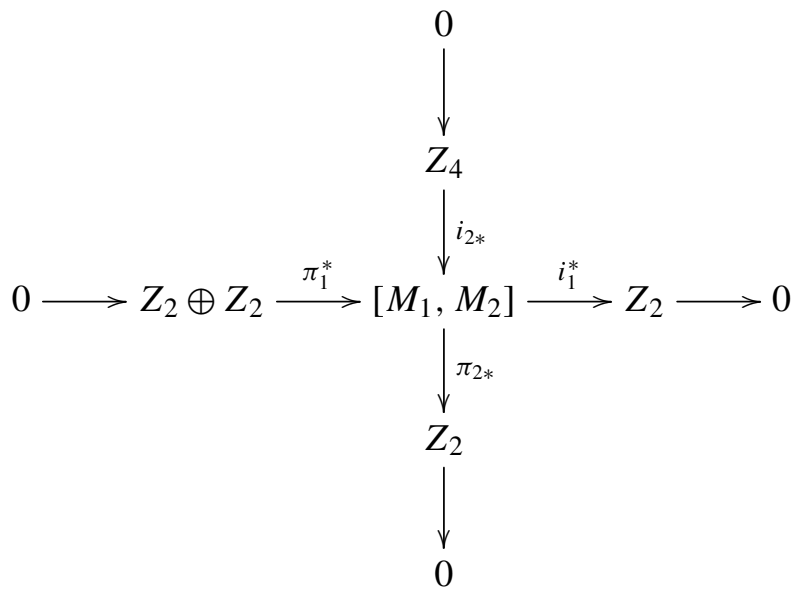

Thus, $\left[M_{1}, M_{2}\right]$ is isomorphic to one of three groups: $Z_{8}, Z_{4} \oplus Z_{2}$ or $Z_{2} \oplus Z_{2} \oplus Z_{2}$. Since $i_{2 *}$ is injective, $\left[M_{1}, M_{2}\right]$ has an element of order 4 . However, $Z_{2} \oplus Z_{2} \oplus Z_{2}$ does not have an element of order 4 . Since $\pi_{1}^{*}$ is injective, $\left[M_{1}, M_{2}\right]$ has a subgroup which is not cyclic. It follows that $\left[M_{1}, M_{2}\right] \neq Z_{8}$. Therefore, $\left[M_{1}, M_{2}\right] \cong Z_{4} \oplus Z_{2}$. 
Now, let $p \equiv 2(\bmod 4)$ and $q \equiv 0(\bmod 4)$. With the results in [Araki and Toda 1965], we obtain the following diagram from the above commutative diagram:

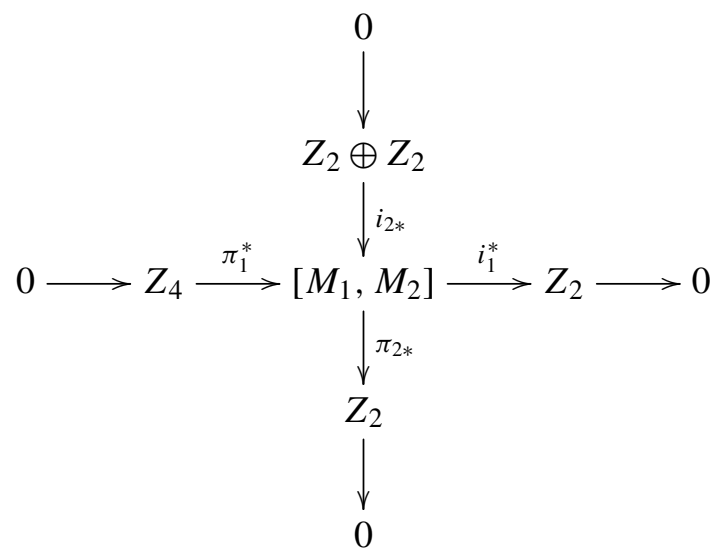

Thus, $\left[M_{1}, M_{2}\right]$ is isomorphic to one of the three groups: $Z_{8}, Z_{4} \oplus Z_{2}$ or $Z_{2} \oplus Z_{2} \oplus Z_{2}$. Since $\pi_{1}^{*}$ is injective, $\left[M_{1}, M_{2}\right]$ has an element of order 4 . However, $Z_{2} \oplus Z_{2} \oplus Z_{2}$ does not have an element of order 4 . Since $i_{2 *}$ is injective, $\left[M_{1}, M_{2}\right]$ has a subgroup which is not cyclic. It follows that $\left[M_{1}, M_{2}\right] \neq Z_{8}$. Thus, $\left[M_{1}, M_{2}\right] \cong Z_{4} \oplus Z_{2}$.

By Lemma 3.4, $\left[M_{1}, M_{2}\right] \cong Z_{4} \oplus Z_{2}$. However, $\left[M_{1}, M_{2}\right]$ has different generators under different conditions. Here we determine the generators.

If $p \equiv 0(\bmod 4)$ and $q \equiv 2(\bmod 4)$, then $\left[M_{1}, M_{2}\right] \cong Z_{4} \oplus Z_{2}\left\{\alpha, \pi_{1}^{*}\left(\eta_{2}\right)\right\}$, where $\pi_{1}^{*}\left(\eta_{1}\right)=2 \alpha$ and $i_{1}^{*}(\alpha)=i_{2 \sharp}(\eta)$.

If $p \equiv 2(\bmod 4)$ and $q \equiv 0(\bmod 4)$, then $\left[M_{1}, M_{2}\right] \cong Z_{4} \oplus Z_{2}\left\{\pi_{1}^{*}(\bar{\eta}), \beta\right\}$, where $i_{1}^{*}(\beta)=i_{2 \sharp}(\eta)$.

For a given homomorphism $h: G_{1} \rightarrow G_{2}$, we have from Proposition 2.6 the commutative diagram

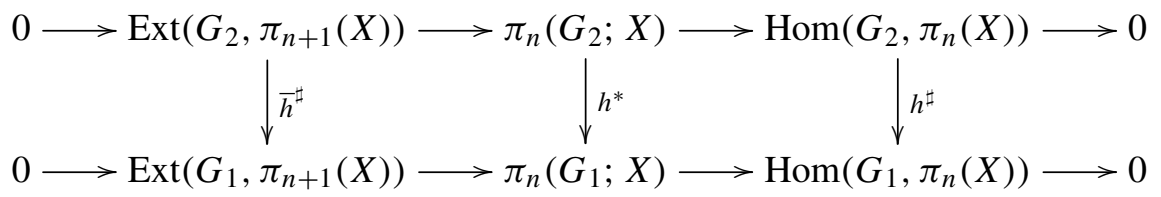

where $\bar{h}^{\sharp}$ and $h^{\sharp}$ are induced by $h$ and $h^{*}$ is associated with $h$. This shows that the nonuniqueness of $h^{*}$ is substantially limited. The measure of choice is bounded by the group

$$
\operatorname{Hom}\left(\operatorname{Hom}\left(G_{2}, \pi_{n}(X)\right), \operatorname{Ext}\left(G_{1}, \pi_{n+1}(X)\right)\right) .
$$

Lemma 3.5. If $(p, q)=d \neq 1$, we have

$$
\left[M_{1}, M_{2}\right] \cong \begin{cases}Z_{2} \oplus Z_{2} & \text { if } p \equiv 2 \text { and } q \equiv 2(\bmod 4) \\ Z_{2} \oplus Z_{2} \oplus Z_{2} & \text { if } p \equiv 0 \text { and } q \equiv 0(\bmod 4) .\end{cases}
$$


Proof. Suppose that $p \equiv 2(\bmod 4)$ and $q \equiv 2(\bmod 4)$. By the universal coefficient theorem for homotopy groups with coefficients, we have the short exact sequence

$$
0 \rightarrow \operatorname{Ext}\left(Z_{q}, Z_{4}\right) \rightarrow\left[M_{1}, M_{2}\right] \rightarrow \operatorname{Hom}\left(Z_{q}, Z_{2}\right) \rightarrow 0 .
$$

Since $\operatorname{Ext}\left(Z_{q}, Z_{4}\right) \cong Z_{(q, 4)} \cong Z_{2}$ and $\operatorname{Hom}\left(Z_{q}, Z_{2}\right)=Z_{(q, 2)}=Z_{2}$, this sequence becomes

$$
0 \rightarrow Z_{2} \rightarrow\left[M_{1}, M_{2}\right] \rightarrow Z_{2} \rightarrow 0
$$

Let $M_{3}=M\left(Z_{p}, n+1\right)$. By the universal coefficient theorem for homotopy with coefficients, we have the sequence

$$
0 \rightarrow \operatorname{Ext}\left(Z_{p}, Z_{4}\right) \rightarrow\left[M_{3}, M_{2}\right] \rightarrow \operatorname{Hom}\left(Z_{p}, Z_{2}\right) \rightarrow 0 .
$$

Similarly, this sequence becomes

$$
0 \rightarrow Z_{2} \rightarrow\left[M_{3}, M_{2}\right] \rightarrow Z_{2} \rightarrow 0 .
$$

We may assume that $q \geq p$. Let $q=k d$ and $p=l d$, where $(k, l)=1$. Then both $k$ and $l$ are odd. We define $h: Z_{q} \rightarrow Z_{p}$ by $h(\overline{1})=\bar{l}$ with $\bar{s}=s+r Z \in Z_{r}$. Then, $\operatorname{im}(h)$ is congruent to $Z_{d}$ in $Z_{p}$ and $h$ is a nontrivial homomorphism since $(q, p)=d \neq 1$. Thus, we have the commutative diagram

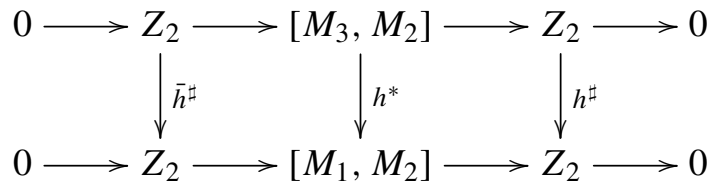

where $\bar{h}^{\sharp}: \operatorname{Ext}\left(Z_{p}, Z_{4}\right) \rightarrow \operatorname{Ext}\left(Z_{q}, Z_{4}\right)$ and $h^{\sharp}: \operatorname{Hom}\left(Z_{p}, Z_{2}\right) \rightarrow \operatorname{Hom}\left(Z_{q}, Z_{2}\right)$ are induced by $h$.

To show that $h^{\sharp}: \operatorname{Hom}\left(Z_{p}, Z_{2}\right) \rightarrow \operatorname{Hom}\left(Z_{q}, Z_{2}\right)$ is an isomorphism, it is sufficient to show that $h^{\sharp}$ is nontrivial. Let $\alpha$ be an nonzero element in $\operatorname{Hom}\left(Z_{p}, Z_{2}\right)$ such that $\alpha(\overline{1})=\overline{1}$. Since $h^{\sharp}(\alpha)=\alpha \circ h \in \operatorname{Hom}\left(Z_{q}, Z_{2}\right)$ and $\alpha \circ h(\overline{1})=\alpha(\bar{l})=\bar{l}=\overline{1}$, where $l$ is odd, it follows that $h^{\sharp}(\alpha)$ is a nontrivial homomorphism.

Next, we show that $\bar{h}^{\sharp}: \operatorname{Ext}\left(Z_{p}, Z_{4}\right) \rightarrow \operatorname{Ext}\left(Z_{q}, Z_{4}\right)$ is an isomorphism. Consider the resolutions of $Z_{q}$ and $Z_{p}$. Then we have following commutative diagram:

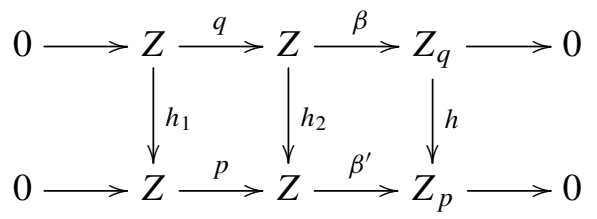

See [Gray 1975, Lemma 25.3]. Now, we give precise definitions of the maps $h_{1}, h_{2}$ and $h^{\sharp}$. Since $\bar{l}=h(\overline{1})=h \circ \beta(1)=\beta^{\prime}\left(h_{2}(1)\right)$, we have $h_{2}$ given by $h_{2}(1)=l$. Moreover, we can obtain $h_{1}$ using $h_{2}$. Since $p \circ h_{1}=h_{2} \circ q$, we have 
$p h_{1}(1)=h_{2}(q)=q h_{2}(1)=d k l=p k$. Thus, $h_{1}$ is given by $h_{1}(1)=k$. If we consider the three homomorphisms $h^{\sharp}, h_{1}^{\sharp}$ and $h_{2}^{\sharp}$ induced by $h, h_{1}$ and $h_{2}$ respectively, we have the following commutative diagram:

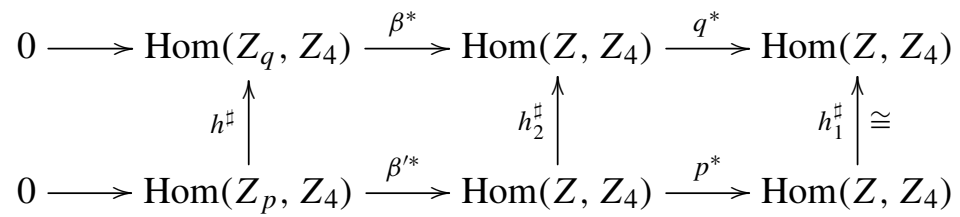

Next, we show that $h_{1}^{\sharp}$ is an isomorphism. We choose a generator $\alpha$ of $\operatorname{Hom}\left(Z, Z_{4}\right)$ such that $\alpha(1)=\overline{1}$. Then $h_{1}^{\sharp}(\alpha)(1)=\left(\alpha \circ h_{1}\right)(1)=\alpha(k) \neq 0(\bmod 2)$ since $k$ is odd. Therefore, $h_{1}^{\sharp}(\alpha)$ is a generator of $\operatorname{Hom}\left(Z, Z_{4}\right)$. Thus, $h_{1}^{\sharp}$ is an isomorphism.

By using $h_{1}^{\sharp}$, we determine the homomorphism $\bar{h}^{\sharp}: \operatorname{Ext}\left(Z_{p}, Z_{4}\right) \rightarrow \operatorname{Ext}\left(Z_{q}, Z_{4}\right)$. Since $q \equiv p \equiv 2(\bmod 4)$ and

$\operatorname{Ext}\left(Z_{p}, Z_{4}\right)=\operatorname{Hom}\left(Z, Z_{4}\right) / \operatorname{im}\left(p^{*}\right)$ and $\operatorname{Ext}\left(Z_{q}, Z_{4}\right)=\operatorname{Hom}\left(Z, Z_{4}\right) / \operatorname{im}\left(q^{*}\right)$, we have

$$
\operatorname{Ext}\left(Z_{p}, Z_{4}\right)=\langle\alpha+\{2 \alpha\}\rangle \quad \text { and } \operatorname{Ext}\left(Z_{q}, Z_{4}\right)=\langle\alpha+\{2 \alpha\}\rangle \text {. }
$$

By well-known facts of homological algebra, $\bar{h}^{\sharp}: \operatorname{Ext}\left(Z_{p}, Z_{4}\right) \rightarrow \operatorname{Ext}\left(Z_{q}, Z_{4}\right)$ is given by $\bar{h}^{\sharp}(\alpha+\{2 \alpha\})=\alpha \circ h_{1}+\{2 \alpha\} \neq 0$. Therefore, $\bar{h}^{\sharp}$ is nontrivial. Thus, $\bar{h}^{\sharp}$ is an isomorphism.

By the five lemma, $h^{*}:\left[M_{1}, M_{2}\right] \rightarrow\left[M_{3}, M_{2}\right]$ is an isomorphism. From [Araki and Toda 1965], we have $\left[M_{3}, M_{2}\right] \cong Z_{2} \oplus Z_{2}$. Therefore, $\left[M_{1}, M_{2}\right] \cong Z_{2} \oplus Z_{2}$.

Next, we suppose that $q \equiv 0$ and $p \equiv 0(\bmod 4)$.

From [Araki and Toda 1965] and the commutative diagram above Lemma 3.4, we obtain the following commutative diagram:

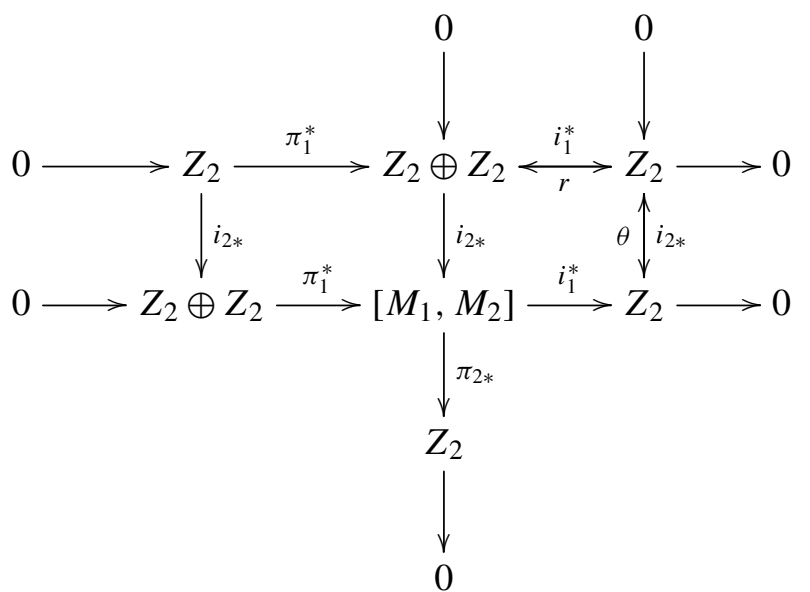


Since the second row is a split exact sequence, there exists $r:\left[S^{n+1}, S^{n}\right] \rightarrow\left[M_{1}, S^{n}\right]$ such that $i_{1}^{*} \circ r=\operatorname{id}_{\left[S^{n+1}, S^{n}\right]}$. Moreover, since the third $i_{2 *}$ is an isomorphism, there exists $\theta:\left[S^{n+1}, M_{2}\right] \rightarrow\left[S^{n+1}, S^{n}\right]$ such that $\theta \circ i_{2 *}=\operatorname{id}_{\left[S^{n+1}, S^{n}\right]}$ and $i_{2 *} \circ \theta=\operatorname{id}_{\left[S^{n+1}, M_{2}\right]}$.

We define the map $k:\left[S^{n+1}, M_{2}\right] \rightarrow\left[M_{1}, M_{2}\right]$ by $k=i_{2 *} \circ r \circ \theta$. Then, we have

$$
\begin{aligned}
i_{1}^{*} \circ k & =i_{1}^{*} \circ i_{2 *} \circ r \circ \theta \\
& =i_{2 *} \circ i_{1}^{*} \circ r \circ \theta \\
& =i_{2 *} \circ \mathrm{id}_{\left[S^{n+1}, S^{n}\right]} \circ \theta \\
& =i_{2 *} \circ \theta=\mathrm{id}_{\left[S^{n+1}, M_{2}\right]} .
\end{aligned}
$$

Therefore, the third row is a split exact sequence. Hence,

$$
\left[M_{1}, M_{2}\right] \cong Z_{2} \oplus Z_{2} \oplus Z_{2} \text {. }
$$

Now, we determine the generators of $\left[M_{1}, M_{2}\right]$ when either $p \equiv 2(\bmod 4)$ and $q \equiv 2(\bmod 4)$ or $p \equiv 0(\bmod 4)$ and $q \equiv 0(\bmod 4)$.

Let $p \equiv 2(\bmod 4)$ and $q \equiv 2(\bmod 4)$. By using the Puppe exact sequence, we have the following exact sequence:

$$
\pi_{n+2}\left(M_{2}\right) \stackrel{q_{\iota_{1}}^{*}}{\longrightarrow} \pi_{n+2}\left(M_{2}\right) \stackrel{\pi_{1}^{*}}{\longrightarrow}\left[M_{1}, M_{2}\right] \stackrel{i_{1}^{*}}{\longrightarrow} \pi_{n+1}\left(M_{2}\right) \stackrel{p_{\iota_{1}}^{*}}{\longrightarrow} \pi_{n+1}\left(M_{2}\right) .
$$

By exactness, we obtain the exact sequence

$$
0 \longrightarrow Z_{2} \stackrel{\pi_{1}^{*}}{\longrightarrow}\left[M_{1}, M_{2}\right] \stackrel{i_{1}^{*}}{\longrightarrow} Z_{2} \longrightarrow 0 .
$$

Thus, $\left[M_{1}, M_{2}\right] \cong Z_{2} \oplus Z_{2}\left\{\pi_{1}^{*}(\bar{\eta}), \beta\right\}$, where $i_{1}^{*}(\beta)=i_{2 \sharp}(\eta)$.

Next, we let $p \equiv 0(\bmod 4)$ and $q \equiv 0(\bmod 4)$. By a similar method we obtain $\left[M_{1}, M_{2}\right] \cong Z_{2} \oplus Z_{2} \oplus Z_{2}\left\{\pi_{1}^{*}\left(\eta_{1}\right), \pi_{1}^{*}\left(\eta_{2}\right), \alpha\right\}$, where $i_{1}^{*}(\alpha)=i_{2 \sharp}(\eta)$.

Remark 3.6. Here we determine the generators of $\pi_{n+3}\left(M\left(Z_{q}, n\right)\right)$. By using the mapping cone sequence of the Moore space

$$
S^{n} \stackrel{q_{\iota}}{\longrightarrow} S^{n} \stackrel{i}{\longrightarrow} M\left(Z_{q}, n\right) \stackrel{\pi}{\longrightarrow} S^{n+1} \stackrel{q_{\iota}}{\longrightarrow} S^{n+1},
$$

we obtain a long exact sequence

$$
\pi_{n+3}\left(S^{n}\right) \stackrel{q_{\text {出}}}{\longrightarrow} \pi_{n+3}\left(S^{n}\right) \stackrel{i_{\sharp}}{\longrightarrow} \pi_{n+3}\left(M\left(Z_{q}, n\right)\right) \stackrel{\pi_{\sharp}}{\longrightarrow} \pi_{n+3}\left(S^{n+1}\right) \stackrel{q_{\text {出}}}{\longrightarrow} \pi_{n+3}\left(S^{n+1}\right) .
$$

From the work by Toda [1962], we have

$$
Z_{24}\{v\} \stackrel{q_{\iota \sharp}}{\longrightarrow} Z_{24}\{v\} \stackrel{i_{\sharp}}{\longrightarrow} \pi_{n+3}\left(M\left(Z_{q}, n\right)\right) \stackrel{\pi_{\sharp}}{\longrightarrow} Z_{2}\left\{\eta^{2}\right\} \stackrel{q_{\iota \sharp}}{\longrightarrow} Z_{2}\left\{\eta^{2}\right\} .
$$

Thus, if $q$ is odd, then $\pi_{n+3}\left(M\left(Z_{q}, n\right)\right) \cong Z_{(q, 24)}\left\{i_{\sharp}(v)\right\}$, and if $q$ is even, then $\pi_{n+3}\left(M\left(Z_{q}, n\right)\right) \cong Z_{(q, 24)} \oplus Z_{2}\left\{i_{\sharp}(v), \overline{\eta^{2}}\right\}$ where $\pi_{\sharp}\left(\overline{\eta^{2}}\right)=\eta^{2}$. 
Based on Remarks 3.1 and 3.6, we obtain for $M_{1}$ the table $q$ odd $\quad q \equiv 2(\bmod 4)$

$$
q \equiv 0(\bmod 4)
$$

$\pi_{n+3}\left(M_{1}\right)$

$Z_{4}$ $Z_{2} \oplus Z_{2}$

Generator

$\hat{\eta}$

Relation

$$
i_{1 \sharp}\left(\eta^{2}\right)=2 \hat{\eta}, \pi_{1 \sharp}(\hat{\eta})=\eta
$$

$$
\begin{gathered}
\eta_{3}, \eta_{4} \\
i_{1 \sharp}\left(\eta^{2}\right)=\eta_{3}, \pi_{1 \sharp}\left(\eta_{4}\right)=\eta
\end{gathered}
$$

while for $M_{2}$ we obtain

$\begin{array}{lccc} & p \text { odd } & p \equiv 2(\bmod 4) & p \equiv 0(\bmod 4) \\ \pi_{n+3}\left(M_{2}\right) & Z_{(p, 24)} & Z_{(p, 24)} \oplus Z_{2} & Z_{(p, 24)} \oplus Z_{2} \\ \text { Generator } & i_{2 \sharp}(v) & i_{2 \sharp}(\nu), \overline{\eta^{2}} & i_{2 \sharp}(v), \overline{\eta^{2}} \\ \text { Relation } & & \pi_{2 \sharp}\left(\overline{\eta^{2}}\right)=\eta^{2} & \pi_{2 \sharp}\left(\overline{\eta^{2}}\right)=\eta^{2}\end{array}$

By Lemmas 3.4 and 3.5, we have the following table, where $\pi_{1}^{*}\left(\eta_{1}\right)=2 \alpha$, $i_{1}^{*}(\alpha)=i_{2 \sharp}(\eta)$ and $i_{1}^{*}(\beta)=i_{2 \sharp}(\eta)$ :

$$
\left[M_{1}, M_{2}\right] \quad \text { Generator }
$$

$\begin{array}{lcc}\text { either } q \text { odd or } p \text { odd } & 0 & \\ q \equiv 2, p \equiv 0(\bmod 4) & Z_{4} \oplus Z_{2} & \alpha, \pi_{1}^{*}\left(\eta_{2}\right) \\ q \equiv 0, p \equiv 2(\bmod 4) & Z_{4} \oplus Z_{2} & \pi_{1}^{*}(\bar{\eta}), \beta \\ q \equiv p \equiv 2(\bmod 4) & Z_{2} \oplus Z_{2} & \pi_{1}^{*}(\bar{\eta}), \beta \\ q \equiv p \equiv 0(\bmod 4) & Z_{2} \oplus Z_{2} \oplus Z_{2} & \pi_{1}^{*}\left(\eta_{1}\right), \pi_{1}^{*}\left(\eta_{2}\right), \alpha\end{array}$

\section{Computation of $\mathcal{E}_{\sharp}^{\operatorname{dim}+r}\left(M\left(Z_{q}, n+1\right) \vee M\left(Z_{p}, n\right)\right)$ for $r=0,1$}

In this section, we compute $\mathcal{E}_{\sharp}^{\operatorname{dim}+r}\left(M_{1} \vee M_{2}\right)$, where $M_{1}=M\left(Z_{q}, n+1\right)=$ $S^{n+1} \cup_{q} e^{n+2}$ and $M_{2}=M\left(Z_{p}, n\right)=S^{n} \cup_{p} e^{n+1}$ with $p, q \geq 1$. In [Jeong 2010], these groups were computed in the case of $p=q$. However, we compute those groups in the general case, that is, $p \neq q$ and $r=0,1$. Throughout this section we assume that $X=M_{1} \vee M_{2}$. Note that $\pi_{n+k}\left(M_{1} \vee M_{2}\right) \cong \pi_{n+k}\left(M_{1}\right) \oplus \pi_{n+k}\left(M_{2}\right)$ for $k \leq n$ by Proposition 2.1. Moreover, from Proposition 2.2, we can identify $f \in[X, X]$ with the $2 \times 2$ matrix

$$
\theta(f)=\left(\begin{array}{ll}
f_{11} & f_{12} \\
f_{21} & f_{22}
\end{array}\right)
$$

where $f_{11} \in\left[M_{1}, M_{1}\right], f_{12} \in\left[M_{2}, M_{1}\right], f_{21} \in\left[M_{1}, M_{2}\right]$, and $f_{22} \in\left[M_{1}, M_{1}\right]$.

Lemma 4.1. Let $f \in[X, X]$ be given by

$$
f=\left(\begin{array}{ll}
f_{11} & f_{12} \\
f_{21} & f_{22}
\end{array}\right) .
$$


Then $f \in \mathcal{E}(X)$ if and only if $f_{11} \in \mathcal{E}\left(M_{1}\right)$ and $f_{22} \in \mathcal{E}\left(M_{2}\right)$. Additionally, if $f \in \mathcal{E}_{\sharp}^{\operatorname{dim}}(X)$, then $f_{22}=1$.

Proof. Let us denote by $h_{* n}: H_{n}(U) \rightarrow H_{n}(V)$ the induced homomorphism on the homology group from $h: U \rightarrow V$. Then, $f \in \mathcal{E}(X)$ if and only if $f_{*}$ is an isomorphism if and only if $f_{11 * n+1}$ and $f_{22 * n}$ are isomorphisms if and only if $f_{11} \in \mathcal{E}\left(M_{1}\right)$ and $f_{22} \in \mathcal{E}\left(M_{2}\right)$. For the proof of the second statement, see [Jeong 2010, Lemma 3.3].

Let us denote by $g_{\sharp s}: \pi_{s}(U) \rightarrow \pi_{s}(V)$ the homomorphism induced by $g: U \rightarrow V$. It is clear from Lemma 4.1 that if $f \in \mathcal{E}(X)$, then $f_{\sharp(n+k)}: \pi_{n+k}(X) \rightarrow \pi_{n+k}(X)$ is given by

$$
f_{\sharp(n+k)}=\left(\begin{array}{ll}
f_{11 \sharp(n+k)} & f_{12 \sharp(n+k)} \\
f_{21 \sharp(n+k)} & f_{22 \sharp(n+k)}
\end{array}\right),
$$

where $f_{11 \sharp(n+k)}$ and $f_{22 \sharp(n+k)}$ are isomorphisms and $k \leq n$.

Lemma 4.2. If $f \in \mathcal{E}(X)$ and either $q$ is odd or $p$ is odd, then $f_{12 \sharp k}=0$ for $k=1,2, \ldots, n+2$.

Proof. Since $M_{1}$ is $n$-connected, we have $\pi_{k}\left(M_{1}\right)=0$ for $k=1,2, \ldots, n$. Thus it is sufficient to show that $f_{12 \sharp k}=0$ for $k=n+1, n+2$.

If $p$ is odd, then $\pi_{n+1}\left(M_{2}\right)$ and $\pi_{n+2}\left(M_{2}\right)$ are trivial groups. Thus, $f_{12 \sharp(n+1)}=$ $f_{12 \sharp(n+2)}=0$.

Suppose that $q$ is odd, $p$ is even and $(p, q)=d \neq 1$. Then, $\pi_{n+1}\left(M_{2}\right) \cong Z_{2}\left\{i_{2 \sharp}(\eta)\right\}$. Since $\left[M_{2}, M_{1}\right] \cong Z_{d}\left\{\pi_{2}^{*}\left(i_{1}\right)\right\}$, we have $f_{12 \sharp n+1}=t \pi_{2}^{*}\left(i_{1}\right)_{\sharp}$ for some integer $t$ such that $1 \leq t \leq d$. Thus, we have

$$
f_{12 \sharp(n+1)}\left(i_{2 \sharp}(\eta)\right)=t \pi_{2}^{*}\left(i_{1}\right)\left(i_{2 \sharp}(\eta)\right)=t\left(i_{1} \circ \pi_{2} \circ i_{2} \circ \eta\right)=0
$$

because $\pi_{2} \circ i_{2}$ is homotopic to a constant map. Hence, $f_{12 \sharp(n+1)}=0$. If $d=1$, $\left[M_{2}, M_{1}\right]=0$ and it is trivial.

For $k=n+2$, we are done since $\pi_{n+2}\left(M_{1}\right)=0$.

Here we introduce certain generators and elements of $\left[M_{1}, M_{1}\right]$ and $\mathcal{E}_{\sharp}^{\operatorname{dim}+r}\left(M_{1}\right)$ for $r=-1,0,1$ as described in [Jeong 2010].

Remark 4.3. Let $M_{1}=M\left(Z_{q}, n+1\right)$ be a Moore space with $q$ is even. By Proposition $2.5, \mathcal{E}_{\sharp}^{\operatorname{dim}}\left(M_{1}\right) \cong Z_{2}$ and $\mathcal{E}_{\sharp}^{\text {dim }+1}\left(M_{1}\right)=1$. In this remark, we describe the generator of $\mathcal{E}_{\sharp}^{\operatorname{dim}}\left(M_{1}\right)$ explicitly.

Consider the mapping cone sequence

$$
S^{n+1} \stackrel{q_{\iota_{1}}}{\longrightarrow} S^{n+1} \stackrel{i_{1}}{\longrightarrow} S^{n+1} \cup_{q} e^{n+2} \stackrel{\pi_{1}}{\longrightarrow} S^{n+2} \stackrel{q_{\iota_{1}}}{\longrightarrow} S^{n+2} .
$$

Then, we have the following exact sequence:

$$
\pi_{n+2}\left(M_{1}\right) \stackrel{q_{l_{1}}^{*}}{\longrightarrow} \pi_{n+2}\left(M_{1}\right) \stackrel{\pi_{1}^{*}}{\longrightarrow}\left[M_{1}, M_{1}\right] \stackrel{i_{1}^{*}}{\longrightarrow} \pi_{n+1}\left(M_{1}\right) \stackrel{q_{l_{1}}^{*}}{\longrightarrow} \pi_{n+1}\left(M_{1}\right) .
$$


Since $\pi_{n+2}\left(M_{1}\right) \cong Z_{2}\left\{i_{1} \eta\right\}$ and $\pi_{n+1}\left(M_{1}\right) \cong Z_{q}\{1\}$, we have the short exact sequence

$$
0 \longrightarrow Z_{2}\left\{i_{1} \eta\right\} \stackrel{\pi_{1}^{*}}{\longrightarrow}\left[M_{1}, M_{1}\right] \stackrel{i_{1}^{*}}{\longrightarrow} Z_{q}\{1\} \longrightarrow 0 .
$$

By [Araki and Toda 1965, Theorem 4.1],

$$
\left[M_{1}, M_{1}\right] \cong \begin{cases}Z_{2 q}\{1\} & \text { if } q \equiv 2(\bmod 4), \\ Z_{q} \oplus Z_{2}\left\{1, i_{1} \circ \eta \circ \pi_{1}\right\} & \text { if } q \equiv 0(\bmod 4),\end{cases}
$$

and

$$
\pi_{1}^{*}\left(i_{1} \circ \eta\right)=i_{1} \circ \eta \circ \pi_{1} \in\left[M_{1}, M_{1}\right] .
$$

Let $i_{1} \circ \eta \circ \pi_{1}=\epsilon$. Then, $\epsilon$ has order 2 and $1+\epsilon \in\left[M_{1}, M_{1}\right]$. Since $n \geq 5$, we have that $1+\epsilon$ is a suspension map. Thus,

$$
(1+\epsilon) \circ(1+\epsilon) \simeq 1 \circ(1+\epsilon)+\epsilon \circ(1+\epsilon)=1+\epsilon+\epsilon+\epsilon \circ \epsilon=1+2 \epsilon+\epsilon^{2} .
$$

If $q \equiv 2(\bmod 4)$, then $i_{1} \circ \eta \circ \pi_{1}=q 1$ and $\epsilon^{2}=i_{1} \circ \eta \circ \pi_{1} \circ i_{1} \circ \eta \circ \pi_{1}$. Since $\pi_{1} \circ i_{1}=0$ and $\epsilon$ has order 2 , we have $2 \epsilon=0$ and $\epsilon^{2}=0$. Thus, $(1+\epsilon) \circ(1+\epsilon) \simeq 1$ and $1+\epsilon \in \mathcal{E}\left(M_{1}\right)$.

Since each $\alpha \in \pi_{n+r}\left(M_{1}\right)$ is a suspension map, for $r=1,2,3$, we have

$$
(1+\epsilon)_{\sharp}(\alpha)=\alpha+\epsilon \circ \alpha .
$$

Since $\pi_{n+1}\left(M_{1}\right) \cong Z_{q}\left\{i_{1}\right\}$ and $\epsilon_{\sharp}\left(i_{1}\right)=i_{1} \circ \eta \circ \pi_{1} \circ i_{1}=0$, we have $1+\epsilon \epsilon$ $\mathcal{E}_{\sharp}^{\operatorname{dim}-1}\left(M_{1}\right)$.

Since $\pi_{n+2}\left(M_{1}\right) \cong Z_{2}\left\{i_{1 \sharp}(\eta)\right\}$ and $\epsilon_{\sharp}\left(i_{1 \sharp}(\eta)\right)=i_{1} \circ \eta \circ \pi_{1} \circ i_{1} \circ \eta=0$, we have $1+\epsilon \in \mathcal{E}_{\sharp}^{\operatorname{dim}}\left(M_{1}\right)$.

Since $\pi_{n+3}\left(M_{1}\right) \cong Z_{4}\{\hat{\eta}\}$ and

$$
\epsilon_{\sharp}(\hat{\eta})=i_{1} \circ \eta \circ \pi_{1} \circ \hat{\eta}=i_{1} \circ \eta \circ \eta=i_{1} \circ \eta^{2}=2 \hat{\eta} \neq 0,
$$

we have $1+\epsilon \notin \mathcal{E}_{\sharp}^{\mathrm{dim}+1}\left(M_{1}\right)$.

We obtain similar results in the case of $q \equiv 0(\bmod 4)$.

Theorem 4.4. If $X=M_{1} \vee M_{2}$ and $(p, q)=1$, then

$$
\mathcal{E}_{\sharp}^{\operatorname{dim}}(X) \cong \begin{cases}1 & \text { if } q \text { is odd }, \\ Z_{2} & \text { if } q \text { is even and } p \text { is odd } .\end{cases}
$$

Proof. Let $(q, p)=1$. Then, either $q$ or $p$ is odd. By Lemmas 3.2 and 3.3, we have $\left[M_{2}, M_{1}\right]=0$ and $\left[M_{1}, M_{2}\right]=0$.

If $q$ is odd, then $\mathcal{E}_{\sharp}^{\operatorname{dim}}\left(M_{1}\right)=1$ and $\mathcal{E}_{\sharp}^{\operatorname{dim}}\left(M_{2}\right)=1$ by Proposition 2.5 and Lemma 4.1. Therefore $\mathcal{E}_{\sharp}^{\operatorname{dim}}(X)=1$. 
If $p$ is odd and $q$ is even, then $\mathcal{E}_{\sharp}^{\operatorname{dim}}\left(M_{1}\right) \cong Z_{2}\{1+\epsilon\}$ and $\mathcal{E}_{\sharp}^{\operatorname{dim}}\left(M_{2}\right)=1$ by Proposition 2.5, Lemma 4.1, and Remark 4.3. Thus, we have

$$
\mathcal{E}_{\sharp}^{\operatorname{dim}}(X) \cong\left\{\left(\begin{array}{cc}
1+\epsilon & 0 \\
0 & 1
\end{array}\right) \mid \epsilon \in Z_{2}\left\{i_{1} \eta \pi_{1}\right\}\right\},
$$

where $\eta$ is the generator of $\pi_{n+2}\left(S^{n+1}\right)$.

Theorem 4.5. If $X=M_{1} \vee M_{2}$ and $(p, q)=d \neq 1$, then

$$
\mathcal{E}_{\sharp}^{\operatorname{dim}}(X) \cong \begin{cases}Z_{d} & \text { if } q \text { is odd, } \\ Z_{2} \oplus Z_{d} & \text { if } q \text { is even and } p \text { is odd. }\end{cases}
$$

Proof. By Lemmas 3.2 and 3.3, we have $\left[M_{2}, M_{1}\right] \cong Z_{d}\left\{\pi_{2}^{*}\left(i_{1}\right)\right\}$ and $\left[M_{1}, M_{2}\right]=0$. Moreover, $f_{12 \sharp k}=0$ for $k=1,2, \ldots, n+2$ by Lemma 4.2.

Thus, if $q$ is odd, then we have

$$
\mathcal{E}_{\sharp}^{\operatorname{dim}}(X) \cong\left\{\left(\begin{array}{cc}
1 & f_{12} \\
0 & 1
\end{array}\right) \mid f_{12} \in Z_{d}\left\{\pi_{2}^{*}\left(i_{1}\right)\right\}\right\},
$$

but if $q$ is even and $p$ is odd, then we have

$$
\mathcal{E}_{\sharp}^{\operatorname{dim}}(X) \cong\left\{\left(\begin{array}{cc}
1+\epsilon & f_{12} \\
0 & 1
\end{array}\right) \mid f_{12} \in Z_{d}\left\{\pi_{2}^{*}\left(i_{1}\right)\right\}, \epsilon \in Z_{2}\left\{i_{1} \eta \pi_{1}\right\}\right\} .
$$

Let $f_{12}$ be an element of $\left[M_{2}, M_{1}\right] \cong Z_{d}\left\{\pi_{2}^{*}\left(i_{1}\right)\right\}$, Then $f_{12}=s \pi_{2}^{*}\left(i_{1}\right)$ for $1 \leq s \leq d$.

Lemma 4.6. For $f=\left(\begin{array}{ll}f_{11} & f_{12} \\ f_{21} & f_{22}\end{array}\right) \in \mathcal{E}(X)$, let $p$ and $q$ be even. Then, $f_{12 \sharp k}=0$ for $k=1,2, \ldots, n+1$.

Proof. Since $M_{1}$ is $n$-connected, $\pi_{k}\left(M_{1}\right)=0$ for $k=1,2, \ldots, n$. Thus, it is sufficient to show that $f_{12 \sharp(n+1)}=0$. Since $\left[M_{2}, M_{1}\right] \cong Z_{d}\left\{\pi_{2}^{*}\left(i_{1}\right)\right\}$ by Lemma 3.2 and $f_{12}$ belongs to $\left[M_{2}, M_{1}\right]$, we have $f_{12}=s \pi_{2}^{*}\left(i_{1}\right)$ for some $1 \leq s \leq d$. Moreover, $\pi_{n+1}\left(M_{2}\right) \cong Z_{2}\left\{i_{2 \sharp}(\eta)\right\}$ by Remark 3.1. Thus, we have

$$
f_{12 \sharp(n+1)}\left(i_{2 \sharp}(\eta)\right)=s \pi_{2}^{*}\left(i_{1}\right)\left(i_{2 \sharp}(\eta)\right)=s\left(i_{1} \circ \pi_{2} \circ i_{2} \circ \eta\right)=0
$$

since $\pi_{2} \circ i_{2}$ is homotopic to the constant map.

Lemma 4.7. Let $p$ and $q$ be even and $f_{12}=s \pi_{2}^{*}\left(i_{1}\right)$ be an element of $\left[M_{2}, M_{1}\right] \cong$ $Z_{d}\left\{\pi_{2}^{*}\left(i_{1}\right)\right\}$ for $1 \leq s \leq d$. Then, $f_{12 \sharp(n+2)} \neq 0$ if $s$ is odd, and $f_{12 \sharp(n+2)}=0$ if $s$ is even.

Proof. First, we note that $\pi_{n+2}\left(M_{1}\right) \cong Z_{2}\left\{i_{\sharp 1}(\eta)\right\}$.

Suppose that $p \equiv 0(\bmod 4)$. Since $\pi_{n+2}\left(M_{2}\right) \cong Z_{2} \oplus Z_{2}\left\{\eta_{1}, \eta_{2}\right\}$, we have

$$
\pi_{2}^{*}\left(i_{1}\right)=\pi_{2}^{*}\left(i_{1}\right)\left(\eta_{1}\right)=\pi_{2}^{*}\left(i_{1}\right)\left(i_{2 \sharp}\left(\eta^{2}\right)\right)=i_{1} \circ \pi_{2} \circ i_{2} \circ \eta^{2}=0
$$

and

$$
\pi_{2}^{*}\left(i_{1}\right)\left(\eta_{2}\right)=i_{1} \circ \pi_{2} \circ \eta_{2}=i_{1} \circ \eta \neq 0 .
$$


Thus, $f_{12 \sharp(n+2)}\left(\eta_{1}\right)=0$ for all $f_{12}$. Moreover, if $s=2 l$ for some $1 \leq l \leq d / 2$, then

$$
s \pi_{2}^{*}\left(i_{1}\right)\left(\eta_{2}\right)=s i_{1} \circ \pi_{2} \circ \eta_{2}=2 l i_{1} \circ \eta=0 .
$$

Therefore, each element in $\left\langle 2 \pi_{2}^{*}\left(i_{1}\right)\right\rangle \cong Z_{d / 2}$ induces the trivial homomorphism on $\pi_{n+2}\left(M_{2}\right)$. However, if $s=2 l+1$ for some $0 \leq l<d / 2-1$, then

$$
s \pi_{2}^{*}\left(i_{1}\right)\left(\eta_{2}\right)=s i_{1} \circ \pi_{2} \circ \eta_{2}=(2 l+1) i_{1} \circ \eta=i_{1} \circ \eta \neq 0 .
$$

Thus, if $f_{12}$ does not belong to $\left\langle 2 \pi_{2}^{*}\left(i_{1}\right)\right\rangle \cong Z_{d / 2}$, then $f_{12 \sharp(n+2)} \neq 0$.

Suppose that $p \equiv 2(\bmod 4)$. Since $\pi_{n+2}\left(M_{2}\right) \cong Z_{4}\{\bar{\eta}\}$, we have

$$
\pi_{2}^{*}\left(i_{1}\right)_{\sharp}(\bar{\eta})=i_{1} \circ \pi_{2} \circ \bar{\eta}=i_{1} \circ \eta=i_{1 \sharp}(\eta) \neq 0 .
$$

If $s=2 k$ for some $1 \leq l \leq d / 2$, then

$$
s \pi_{2}^{*}\left(i_{1}\right)_{\sharp}(\bar{\eta})=s i_{1} \circ \pi_{2} \circ \bar{\eta}=s i_{1} \circ \eta=2 l i_{1 \sharp}(\eta)=0 .
$$

Thus, each element in $\left\langle 2 \pi_{2}^{*}\left(i_{1}\right)\right\rangle \cong Z_{d / 2}$ induces the trivial homomorphism on $n+2$.

However, if $s=2 l+1$ for some $0 \leq l \leq d / 2-1$, then

$$
s \pi_{2}^{*}\left(i_{1}\right)_{\sharp}(\bar{\eta})=s i_{1} \circ \pi_{2} \circ \bar{\eta}=s i_{1} \circ \eta=(2 l+1) i_{1 \sharp}(\eta)=i_{1 \sharp}(\eta) \neq 0 .
$$

Thus if $f_{12}$ does not belong to $\left\langle 2 \pi_{2}^{*}\left(i_{1}\right)\right\rangle \cong Z_{d / 2}$, then $f_{12 \sharp(n+2)} \neq 0$.

Theorem 4.8. Let $p$ and $q$ be even and let $X=M_{1} \vee M_{2}$. Then if $(p, q)=d \neq 1$, we have

$$
\mathcal{E}_{\sharp}^{\operatorname{dim}}(X) \cong \begin{cases}Z_{2} \oplus Z_{d / 2} \oplus Z_{2} \oplus Z_{2} & \text { if } q \equiv 2, p \equiv 0(\bmod 4), \\ Z_{2} \oplus Z_{d / 2} \oplus Z_{4} & \text { if } q \equiv 0, p \equiv 2(\bmod 4), \\ Z_{2} \oplus Z_{d / 2} \oplus Z_{2} & \text { if } q \equiv 2, p \equiv 2(\bmod 4), \\ Z_{2} \oplus Z_{d / 2} \oplus Z_{2} \oplus Z_{2} & \text { if } q \equiv 0, p \equiv 0(\bmod 4) .\end{cases}
$$

Proof. By Proposition $2.5, \mathcal{E}_{\sharp}^{\operatorname{dim}}\left(M_{1}\right) \cong Z_{2}$ and $\mathcal{E}_{\sharp}^{\operatorname{dim}}\left(M_{2}\right)=1$. By Lemma 4.6, for each $f=\left(\begin{array}{ll}f_{11} & f_{12} \\ f_{21} & f_{22}\end{array}\right) \in \mathcal{E}(X)$, we have $f_{12 \sharp k}=0$ for $k=1,2, \ldots, n+1$. By Lemma 4.7, each element in $\left\langle 2 \pi_{2}^{*}\left(i_{1}\right)\right\rangle \cong Z_{d / 2}$ induces the trivial homomorphism on $\pi_{n+2}\left(M_{2}\right)$. Furthermore, if $f_{12}$ does not belong to $\left\langle 2 \pi_{2}^{*}\left(i_{1}\right)\right\rangle \cong Z_{d / 2}$, then $f_{12 \sharp(n+2)} \neq 0$. Thus, it is sufficient to investigate $f_{21 \sharp n}, f_{21 \sharp(n+1)}$ and $f_{21 \sharp(n+2)}$.

Case 1. Let $q \equiv 2(\bmod 4)$ and $p \equiv 0(\bmod 4)$. From Lemma 3.4, we obtain $\left[M_{1}, M_{2}\right] \cong Z_{4} \oplus Z_{2}\left\{\alpha, \pi_{1}^{*}\left(\eta_{2}\right)\right\}$, where $\pi_{1}^{*}\left(\eta_{1}\right)=2 \alpha$ and $i_{1}^{*}(\alpha)=i_{2 \sharp}(\eta)$.

Since $M_{1}$ is n-connected, $\pi_{n}\left(M_{1}\right)=0$. Thus, $f_{21 \sharp n}=0$.

Since $\pi_{n+1}\left(M_{1}\right) \cong Z_{q}\left\{i_{1}\right\}$, we have $\pi_{1}^{*}\left(\eta_{2}\right)_{\sharp}\left(i_{1}\right)=\eta_{2} \circ \pi_{1} \circ i_{1}=0$.

Conversely, since $\pi_{n+1}\left(M_{2}\right) \cong Z_{2}\left\{i_{2 \sharp}(\eta)\right\}$ and $\alpha_{\sharp}\left(i_{1}\right)=\alpha \circ i_{1}=i_{2 \sharp}(\eta) \neq 0$, we have $(2 \alpha)_{\sharp}=0$ and $(3 \alpha)_{\sharp} \neq 0$. Moreover, since $\pi_{n+2}\left(M_{1}\right) \cong Z_{2}\left\{i_{1 \sharp}(\eta)\right\}$, we have $\pi_{1}^{*}\left(\eta_{2}\right)_{\sharp}\left(i_{1 \sharp}(\eta)\right)=\eta_{2} \circ \pi_{1} \circ i_{1} \circ \eta=0$. Hence, $\left(\begin{array}{cc}1 & 0 \\ f_{21} & 1\end{array}\right)$ belongs to $\mathcal{E}_{\sharp}^{\operatorname{dim}}(X)$ if $f_{21} \in Z_{2} \oplus Z_{2}\left\{2 \alpha, \pi_{1}^{*}\left(\eta_{2}\right)\right\}$. 
Therefore,

$$
\mathcal{E}_{\sharp}^{\operatorname{dim}}(X) \cong\left\{\left(\begin{array}{cc}
1+\epsilon & f_{12} \\
f_{21} & 1
\end{array}\right) \mid f_{12} \in\left\langle 2 \pi_{2}^{*}\left(i_{1}\right)\right\rangle, f_{21} \in\langle 2 \alpha\rangle \oplus\left\langle\pi_{1}^{*}\left(\eta_{2}\right)\right\rangle\right\},
$$

where $\epsilon \in\left\langle i_{1} \eta \pi_{1}\right\rangle$.

Case 2. Let $q \equiv 0(\bmod 4)$ and $p \equiv 2(\bmod 4)$. From Lemma 3.4, we obtain $\left[M_{1}, M_{2}\right] \cong Z_{4} \oplus Z_{2}\left\{\pi_{1}^{*}(\bar{\eta}), \beta\right\}$, where $i_{1}^{*}(\beta)=i_{2 \sharp}(\eta)$.

Since $\pi_{n}\left(M_{1}\right)=0$, we have $f_{21 \sharp n}=0$. However, since $\pi_{n+1}\left(M_{1}\right) \cong Z_{q}\left\{i_{1}\right\}$ and $\pi_{n+1}\left(M_{2}\right) \cong Z_{2}\left\{i_{2 \sharp}(\eta)\right\}$, we have $\pi_{1}^{*}(\bar{\eta})_{\sharp}\left(i_{1}\right)=\bar{\eta} \circ \pi_{1} \circ i_{1}=0$, but $\beta_{\sharp}\left(i_{1}\right)=\beta \circ i_{1}=$ $i_{2 \sharp}(\eta) \neq 0$.

For the generator $\pi_{1}^{*}(\bar{\eta})$ of $\left[M_{1}, M_{2}\right] \cong Z_{4} \oplus Z_{2}\left\{\pi_{1}^{*}(\bar{\eta}), \beta\right\}$ and the generator $i_{1 \sharp}(\eta)$ of $\pi_{n+2}\left(M_{1}\right) \cong Z_{2}\left\{i_{1 \sharp}(\eta)\right\}$, we have $\pi_{1}^{*}(\bar{\eta})_{\sharp}\left(i_{1 \sharp}(\eta)\right)=\bar{\eta} \circ \pi_{1} \circ i_{1 \sharp}(\eta)=0$.

Hence, $\left(\begin{array}{cc}1 & 0 \\ f_{21} & 1\end{array}\right)$ belongs to $\mathcal{E}_{\sharp}^{\operatorname{dim}}(X)$ if $f_{21} \in\left\langle\pi_{1}^{*}(\bar{\eta})\right\rangle$.

Therefore,

$$
\mathcal{E}_{\sharp}^{\operatorname{dim}}(X) \cong\left\{\left(\begin{array}{cc}
1+\epsilon & f_{12} \\
f_{21} & 1
\end{array}\right) \mid f_{12} \in\left\langle 2 \pi_{2}^{*}\left(i_{1}\right)\right\rangle, f_{21} \in\left\langle\pi_{1}^{*}(\bar{\eta})\right\rangle\right\},
$$

where $\epsilon \in\left\langle i_{1} \eta \pi_{1}\right\rangle$.

Case 3 . Let $q \equiv 2(\bmod 4)$ and $p \equiv 2(\bmod 4)$. From Lemma 3.5 , we obtain $\left[M_{1}, M_{2}\right] \cong Z_{2} \oplus Z_{2}\left\{\pi_{1}^{*}(\bar{\eta}), \beta\right\}$, where $i_{1}^{*}(\beta)=i_{2 \sharp}(\eta)$.

First, we recall that $f_{21 \sharp n}=0$ since $\pi_{n}\left(M_{1}\right)=0$.

Since $\pi_{n+1}\left(M_{1}\right) \cong Z_{q}\left\{i_{1}\right\}$ and $\pi_{n+1}\left(M_{2}\right) \cong Z_{2}\left\{i_{2 \sharp}(\eta)\right\}$, we have $\pi_{1}^{*}(\bar{\eta})_{\sharp}\left(i_{1}\right)=$ $\bar{\eta} \circ \pi_{1} \circ i_{1}=0$, but $\beta_{\sharp}\left(i_{1}\right)=\beta \circ i_{1}=i_{2 \sharp}(\eta) \neq 0$. Moreover, since $\pi_{n+2}\left(M_{1}\right) \cong$ $Z_{2}\left\{i_{1 \sharp}(\eta)\right\}$, we have $\pi_{1}^{*}(\bar{\eta})_{\sharp}\left(i_{1 \sharp}(\eta)\right)=\bar{\eta} \circ \pi_{1} \circ i_{1} \circ \eta=0$.

Hence, if $f_{21} \in\left\langle\pi_{1}^{*}(\bar{\eta})\right\rangle$, then $\left(\begin{array}{cc}1 & 0 \\ f_{21} & 1\end{array}\right)$ belongs to $\mathcal{E}_{\sharp}^{\operatorname{dim}}(X)$. However, if $f_{21} \in\langle\beta\rangle$, this cannot be the case. Therefore,

$$
\mathcal{E}_{\sharp}^{\operatorname{dim}}(X) \cong\left\{\left(\begin{array}{cc}
1+\epsilon & f_{12} \\
f_{21} & 1
\end{array}\right) \mid f_{12} \in\left\langle 2 \pi_{2}^{*}\left(i_{1}\right)\right\rangle, f_{21} \in\left\langle\pi_{1}^{*}(\bar{\eta})\right\rangle\right\},
$$

where $\epsilon \in\left\langle i_{1} \eta \pi_{1}\right\rangle$.

Case 4. Let $q \equiv 0(\bmod 4)$ and $p \equiv 0(\bmod 4)$. From Lemma 3.5, we obtain $\left[M_{1}, M_{2}\right] \cong Z_{2} \oplus Z_{2} \oplus Z_{2}\left\{\pi_{1}^{*}\left(\eta_{1}\right), \pi_{1}^{*}\left(\eta_{2}\right), \alpha\right\}$, where $i_{1}^{*}(\alpha)=i_{2 \sharp}(\eta)$. First, we note that $f_{21 \sharp n}=0$ since $\pi_{n}\left(M_{1}\right)=0$.

Since $\pi_{n+1}\left(M_{1}\right) \cong Z_{q}\left\{i_{1}\right\}$ and $\pi_{n+1}\left(M_{2}\right) \cong Z_{2}\left\{i_{2 \sharp}(\eta)\right\}$, we have $\pi_{1}^{*}\left(\eta_{1}\right)_{\sharp}\left(i_{1}\right)=$ $\eta_{1} \circ \pi_{1} \circ i_{1}=0$ and $\pi_{1}^{*}\left(\eta_{2}\right)_{\sharp}\left(i_{1}\right)=\eta_{2} \circ \pi_{1} \circ i_{1}=0$, but $\alpha_{\sharp}\left(i_{1}\right)=\alpha \circ i_{1}=i_{2 \sharp}(\eta) \neq 0$. Also, since $\pi_{n+2}\left(M_{1}\right) \cong Z_{2}\left\{i_{1 \sharp}(\eta)\right\}$, we have $\pi_{1}^{*}\left(\eta_{1}\right)_{\sharp}\left(i_{1 \sharp}(\eta)\right)=\eta_{1} \circ \pi_{1} \circ i_{1 \sharp}(\eta)=0$ and $\pi_{1}^{*}\left(\eta_{2}\right)_{\sharp}\left(i_{1 \sharp}(\eta)\right)=\eta_{2} \circ \pi_{1} \circ i_{1 \sharp}(\eta)=0$.

Hence, if $f_{21} \in\left\langle\pi_{1}^{*}\left(\eta_{1}\right)\right\rangle \oplus\left\langle\pi_{1}^{*}\left(\eta_{2}\right)\right\rangle$, then $\left(\begin{array}{rr}1 & 0 \\ f_{21} & 1\end{array}\right)$ belongs to $\mathcal{E}_{\sharp}^{\operatorname{dim}}(X)$. However, if $f_{21} \in\langle\alpha\rangle$, this cannot be the case. Therefore, 


$$
\mathcal{E}_{\sharp}^{\operatorname{dim}}(X) \cong\left\{\left(\begin{array}{cc}
1+\epsilon & f_{12} \\
f_{21} & 1
\end{array}\right) \mid f_{12} \in\left\langle 2 \pi_{2}^{*}\left(i_{1}\right)\right\rangle, f_{21} \in \pi_{1}^{*}\left(\left\langle\eta_{1}\right\rangle \oplus\left\langle\eta_{2}\right\rangle\right)\right\},
$$

where $\epsilon \in\left\langle i_{1} \eta \pi_{1}\right\rangle$.

From Theorems 4.4-4.8, we obtain Table 1 (see page 37).

Theorem 4.9. Let $X=M_{1} \vee M_{2}, n \geq 5$ and $(q, p)=d$. Then we have

$$
\mathcal{E}_{\sharp}^{\operatorname{dim}+1}(X) \cong \begin{cases}1 & \text { if } q \text { is odd or } p \text { is odd }(d=1), \\ Z_{d} & \text { if } q \text { is odd or } p \text { is odd }(d \neq 1), \\ Z_{d / 2} \oplus Z_{2} & \text { if } p \equiv 0(\bmod 4) \text { and }(p, 24)=4 \text { or } 12(d \neq 1), \\ Z_{d / 2} & \text { if } p \equiv 0(\bmod 4) \text { and }(p, 24)=8 \text { or } 24(d \neq 1), \\ Z_{d / 2} & \text { if } q \equiv 2, p \equiv 2(\bmod 4), \\ Z_{d / 2} \oplus Z_{2} & \text { if } q \equiv 0, p \equiv 2(\bmod 4) .\end{cases}
$$

Proof. By virtue of Remark 4.3, Theorem 4.4 and the fact that $\mathcal{E}_{\sharp}^{\operatorname{dim}+1}(X) \subseteq \mathcal{E}_{\sharp}^{\operatorname{dim}}(X)$, we have $\mathcal{E}_{\sharp}^{\operatorname{dim}+1}(X)=1$ if $(p, q)=1$.

By Proposition 2.5, we have $\mathcal{E}_{\sharp}^{\operatorname{dim}+1}\left(M_{1}\right)=1$. Thus, it is sufficient to identify $f_{12 \sharp(n+3)}$ and $f_{21 \sharp(n+3)}$. First, we note that $\left[M_{2}, M_{1}\right] \cong Z_{d}\left\{\pi_{2}^{*}\left(i_{1}\right)\right\}$ by Lemma 3.2. Case 1. Suppose that $q$ is odd or $p$ is odd and $(p, q)=d \neq 1$. Since $\left[M_{1}, M_{2}\right]=0$ by Lemma 3.3, we only investigate $f_{12 \sharp(n+3)}$.

If $q$ is odd, $f_{12 \sharp(n+3)}=0$ since $\pi_{n+3}\left(M_{1}\right)=0$. If $q$ is even and $p$ is odd, $\pi_{n+3}\left(M_{2}\right) \cong Z_{(p, 24)}\left\{i_{2 \sharp}(v)\right\}$. Since

$$
\pi_{2}^{*}\left(i_{1}\right)_{\sharp}\left(i_{2 \sharp}(v)\right)=i_{1} \circ \pi_{2} \circ i_{2} \circ v=0,
$$

we have $f_{12 \sharp(n+3)}=0$ for each $f_{12} \in\left[M_{2}, M_{1}\right]$. Therefore,

$$
\mathcal{E}_{\sharp}^{\operatorname{dim}+1}(X) \cong\left\{\left(\begin{array}{cc}
1 & f_{12} \\
0 & 1
\end{array}\right) \mid f_{12} \in\left\langle\pi_{2}^{*}\left(i_{1}\right)\right\rangle\right\} .
$$

Case 2. Suppose that $q \equiv 2(\bmod 4)$ and $p \equiv 0(\bmod 4)$. First, we note that

$$
\pi_{n+3}\left(M_{2}\right) \cong Z_{(p, 24)} \oplus Z_{2}\left\{i_{2 \sharp}(\nu), \overline{\eta^{2}}\right\}
$$

and that $\pi_{n+3}\left(M_{1}\right) \cong Z_{4}\{\hat{\eta}\}$ by Proposition 2.3. Let $f_{12}=s \pi_{2}^{*}\left(i_{1}\right)$. If $s=2 l$ for some $1 \leq l \leq d / 2$, then

$$
s \pi_{2}^{*}\left(i_{1}\right)_{\sharp}\left(\overline{\eta^{2}}\right)=2 l \pi_{2}^{*}\left(i_{1}\right)_{\sharp}\left(\overline{\eta^{2}}\right)=4 l \hat{\eta}=0
$$

since

$$
\pi_{2}^{*}\left(i_{1}\right)_{\sharp}\left(i_{2 \sharp}(v)\right)=i_{1} \circ \pi_{2} \circ i_{2} \circ v=0
$$

and

$$
\pi_{2}^{*}\left(i_{1}\right)_{\sharp}\left(\overline{\eta^{2}}\right)=i_{1} \circ \pi_{2} \circ \overline{\eta^{2}}=i_{1 \sharp}\left(\eta^{2}\right)=2 \hat{\eta} \neq 0 \in \pi_{n+3}\left(M_{1}\right) \cong Z_{4} .
$$


Further, if $s=2 l+1$ for some $0 \leq l \leq d / 2-1$, then

$$
s \pi_{2}^{*}\left(i_{1}\right)_{\sharp}\left(\overline{\eta^{2}}\right)=(2 l+1) \pi_{2}^{*}\left(i_{1}\right)_{\sharp}\left(\overline{\eta^{2}}\right)=4 l \hat{\eta}+2 \hat{\eta}=2 \hat{\eta} \neq 0 .
$$

Thus, each $f_{12} \in\left\langle 2 \pi_{2}^{*}\left(i_{1}\right)\right\rangle \cong Z_{d / 2}$ induces the trivial homomorphism on $\pi_{n+3}\left(M_{2}\right)$. However, if $f_{12}$ does not belong to $\left\langle 2 \pi_{2}^{*}\left(i_{1}\right)\right\rangle$, then $f_{12 \sharp(n+3)} \neq 0$.

Let us investigate $f_{21 \sharp(n+3)}$. Note that $\left[M_{1}, M_{2}\right] \cong Z_{4} \oplus Z_{2}\left\{\alpha, \pi_{1}^{*}\left(\eta_{2}\right)\right\}$ and $\pi_{n+3}\left(M_{1}\right) \cong Z_{4}\{\hat{\eta}\}$ with $\pi_{1}^{*}\left(\eta_{1}\right)=2 \alpha, i_{1}^{*}(\alpha)=i_{2 \sharp}(\eta), i_{1 \sharp}\left(\eta^{2}\right)=2 \hat{\eta}$ and $\pi_{1 \sharp}(\hat{\eta})=\eta$. Since $\pi_{2 \sharp}\left(\eta_{2} \circ \eta\right)=\eta^{2}$, we have

$$
\pi_{1}^{*}\left(\eta_{2}\right)_{\sharp}(\hat{\eta})=\eta_{2} \circ \pi_{1} \circ \hat{\eta}=\eta_{2} \circ \eta \neq 0 .
$$

Moreover, since $\eta^{3}=4 v$ [Toda 1962, (5.5)], we have

$$
2 \alpha_{\sharp}(\hat{\eta})=2 \alpha \circ \hat{\eta}=\eta_{1} \circ \pi_{1} \circ \hat{\eta}=\eta_{1} \circ \eta=i_{1 \sharp}\left(\eta^{2}\right) \circ \eta=i_{2} \circ \eta^{3}=4 i_{2 \sharp}(\nu) .
$$

Therefore, $\alpha_{\sharp}(\hat{\eta})=2 i_{2 \sharp}(v)$. Since $(p, 24)$ is a multiple of 4 , we have $\alpha_{\sharp}(\hat{\eta})=$ $2 i_{2 \sharp}(v) \neq 0$ and $3 \alpha_{\sharp}(\hat{\eta})=6 i_{2 \sharp}(v) \neq 0$.

Since $v$ is 2-primary, if $(p, 24)=4$ or $(p, 24)=12$, then $2 \alpha_{\sharp}(\hat{\eta})=0$, and if $(p, 24)=8$ or $(p, 24)=24$, then $2 \alpha_{\sharp}(\hat{\eta}) \neq 0$. Thus, each $f_{21} \in\langle 2 \alpha\rangle$ induces the trivial homomorphism on $\pi_{n+3}\left(M_{1}\right)$ provided that $(p, 24)=4$ or $(p, 24)=12$.

Therefore, if $(p, 24)=4$ or $(p, 24)=12$, we have

$$
\mathcal{E}_{\sharp}^{\operatorname{dim}+1}(X) \cong\left\{\left(\begin{array}{cc}
1 & f_{12} \\
f_{21} & 1
\end{array}\right) \mid f_{12} \in\left\langle 2 \pi_{2}^{*}\left(i_{1}\right)\right\rangle, f_{21} \in\langle 2 \alpha\rangle\right\},
$$

and if $(p, 24)=8$ or 24 , we have

$$
\mathcal{E}_{\sharp}^{\operatorname{dim}+1}(X) \cong\left\{\left(\begin{array}{cc}
1 & f_{12} \\
1 & 1
\end{array}\right) \mid f_{12} \in\left\langle 2 \pi_{2}^{*}\left(i_{1}\right)\right\rangle\right\} .
$$

Case 3. Suppose that $q \equiv 0(\bmod 4)$ and $p \equiv 2(\bmod 4)$. We note that

$$
\begin{aligned}
& \pi_{n+3}\left(M_{2}\right) \cong Z_{(p, 24)} \oplus Z_{2}\left\{i_{2 \sharp}(v), \overline{\eta^{2}}\right\}, \\
& \pi_{n+3}\left(M_{1}\right) \cong Z_{2} \oplus Z_{2}\left\{\eta_{3}, \eta_{4}\right\}
\end{aligned}
$$

and $\left[M_{1}, M_{2}\right] \cong Z_{4} \oplus Z_{2}\left\{\pi_{1}^{*}(\bar{\eta}), \beta\right\}$. First, we investigate $f_{12 \sharp(n+3)}$. Let $f_{12}=$ $s \pi_{2}^{*}\left(i_{1}\right) \in\left[M_{2}, M_{1}\right] \cong Z_{d}\left\{\pi_{2}^{*}\left(i_{1}\right)\right\}$. Then, we have

$$
\pi_{2}^{*}\left(i_{1}\right)_{\sharp}\left(i_{2 \sharp}(v)\right)=i_{1} \circ \pi_{2} \circ i_{2} \circ v=0
$$

and

$$
\pi_{2}^{*}\left(i_{1}\right)_{\sharp}\left(\overline{\eta^{2}}\right)=i_{1} \circ \pi_{2} \circ \overline{\eta^{2}}=i_{1} \circ \eta^{2} \neq 0 .
$$

If $s=2 l$ for some $1 \leq l \leq d / 2$, then $2 l \pi_{2}^{*}\left(i_{1}\right)_{\sharp}\left(\overline{\eta^{2}}\right)=2 l i_{1} \circ \eta^{2}=0$, because $i_{1} \circ \eta^{2}=\eta_{3} \in \pi_{n+3}\left(M_{1}\right)$. However, if $s=2 l+1$ for some $0 \leq l \leq d / 2-1$, then $(2 l+1) \pi_{2}^{*}\left(i_{1}\right)_{\sharp}\left(\overline{\eta^{2}}\right)=(2 k+1) i_{1} \circ \eta^{2}=i_{1} \circ \eta^{2} \neq 0$.

Thus, any $f_{12} \in\left\langle 2 \pi_{2}^{*}\left(i_{1}\right)\right\rangle \cong Z_{d / 2}$ induces the trivial homomorphism on $\pi_{n+3}\left(M_{2}\right)$. However, for $f_{12} \notin\left\langle 2 \pi_{2}^{*}\left(i_{1}\right)\right\rangle$, we have $f_{12 \sharp n+3} \neq 0$. 
Next, we investigate $f_{21 \sharp(n+3)}$. Because $\left[M_{1}, M_{2}\right] \cong Z_{4} \oplus Z_{2}\left\{\pi_{1}^{*}(\bar{\eta}), \beta\right\}$ and $\beta_{\sharp(n+2)} \neq 0$, we check only the generators $\pi_{1}^{*}(\bar{\eta})$. For $\eta_{3}$, we have

$$
\pi_{1}^{*}(\bar{\eta})_{\sharp}\left(\eta_{3}\right)=\bar{\eta} \circ \pi_{1} \circ \eta_{3}=\bar{\eta} \circ \pi_{1} \circ i_{1 \sharp}\left(\eta^{2}\right)=0 .
$$

For $\eta_{4}$, we have

$$
\pi_{1}^{*}(\bar{\eta})_{\sharp}\left(\eta_{4}\right)=\bar{\eta} \circ \pi_{1} \circ \eta_{4}=\bar{\eta} \circ \eta \neq 0
$$

since $\pi_{2 \sharp}(\bar{\eta} \circ \eta)=\eta^{2} \neq 0$.

However, $2 \pi_{1}^{*}(\bar{\eta})_{\sharp}\left(\eta_{4}\right)=\bar{\eta} \circ \pi_{1} \circ 2 \eta_{4}=0$.

Thus, every $f_{21} \in\left\langle 2 \pi_{1}^{*}(\bar{\eta})\right\rangle$ induces the trivial homomorphism on $n+3$.

Therefore, we have

$$
\mathcal{E}_{\sharp}^{\mathrm{dim}+1}(X) \cong\left\{\left(\begin{array}{cc}
1 & f_{12} \\
f_{21} & 1
\end{array}\right) \mid f_{12} \in\left\langle 2 \pi_{2}^{*}\left(i_{1}\right)\right\rangle, f_{21} \in\left\langle 2 \pi_{1}^{*}(\bar{\eta})\right\rangle\right\} .
$$

Case 4. Suppose that $q \equiv 2(\bmod 4)$ and $p \equiv 2(\bmod 4)$. Note that $\pi_{n+3}\left(M_{2}\right) \cong$ $Z_{(p, 24)} \oplus Z_{2}\left\{i_{2 \sharp}(v), \overline{\eta^{2}}\right\}$ and $\pi_{n+3}\left(M_{1}\right) \cong Z_{4}\{\hat{\eta}\}$. First, we investigate $f_{12 \sharp(n+3)}$. For the generator $\pi_{2}^{*}\left(i_{1}\right)$ of $\left[M_{2}, M_{1}\right]$, we have

$$
\pi_{2}^{*}\left(i_{1}\right)_{\sharp}\left(i_{2 \sharp}(v)\right)=i_{1} \circ \pi_{2} \circ i_{2} \circ v=0
$$

and

$$
\pi_{2}^{*}\left(i_{1}\right)_{\sharp}\left(\overline{\eta^{2}}\right)=i_{1} \circ \pi_{2} \circ \overline{\eta^{2}}=i_{1} \circ \eta^{2}=2 \hat{\eta} \neq 0 .
$$

Let $f_{12}=s \pi_{2}^{*}\left(i_{1}\right)$. If $s=2 l$ for $1 \leq l \leq d / 2$, then $s \pi_{2}^{*}\left(i_{1}\right)_{\sharp}\left(\overline{\eta^{2}}\right)=4 l \hat{\eta}=0$, and if $s=2 l+1$ for $0 \leq l \leq d / 2-1$, then $s \pi_{2}^{*}\left(i_{1}\right)_{\sharp}\left(\overline{\eta^{2}}\right)=(4 l+2) \hat{\eta}=2 \hat{\eta} \neq 0$.

Thus, each $f_{12} \in\left\langle 2 \pi_{2}^{*}\left(i_{1}\right)\right\rangle \cong Z_{d / 2}$ induces the trivial homomorphism on $n+3$. However, for $f_{12} \notin\left\langle 2 \pi_{2}^{*}\left(i_{1}\right)\right\rangle$, we have $f_{12 \sharp(n+3)} \neq 0$.

Next, we investigate $f_{21 \sharp(n+3)}$. Note that $\left[M_{1}, M_{2}\right] \cong Z_{2} \oplus Z_{2}\left\{\pi_{1}^{*}(\bar{\eta}), \beta\right\}$. Since $\beta_{\sharp n+2} \neq 0$, we consider only the generator $\pi_{1}^{*}(\bar{\eta})$.

Since $\pi_{2 \sharp}(\bar{\eta} \circ \eta)=\pi_{2} \circ \bar{\eta} \circ \eta=\eta^{2} \neq 0$, we have $\pi_{1}^{*}(\bar{\eta})_{\sharp}(\hat{\eta})=\bar{\eta} \circ \pi_{1} \circ \hat{\eta}=\bar{\eta} \circ \eta \neq 0$. Therefore, no $f_{21}$ induces a trivial homomorphism.

Thus, we have

$$
\mathcal{E}_{\sharp}^{\operatorname{dim}+1}(X) \cong\left\{\left(\begin{array}{cc}
1 & f_{12} \\
1 & 1
\end{array}\right) \mid f_{12} \in\left\langle 2 \pi_{2}^{*}\left(i_{1}\right)\right\rangle\right\} .
$$

Case 5. Suppose that $q \equiv 0(\bmod 4)$ and $p \equiv 0(\bmod 4)$. Note that $\pi_{n+3}\left(M_{2}\right) \cong$ $Z_{(p, 24)} \oplus Z_{2}\left\{i_{2 \sharp}(v), \overline{\eta^{2}}\right\}$ and $\pi_{n+3}\left(M_{1}\right) \cong Z_{2} \oplus Z_{2}\left\{\eta_{3}, \eta_{4}\right\}$.

First, we investigate $f_{12 \sharp(n+3)}$. For the generator $\pi_{2}^{*}\left(i_{1}\right)$ of $\left[M_{2}, M_{1}\right]$, we have

$$
\pi_{2}^{*}\left(i_{1}\right)_{\sharp}\left(i_{2 \sharp}(v)\right)=i_{1} \circ \pi_{2} \circ i_{2} \circ v=0
$$

and

$$
\pi_{2}^{*}\left(i_{1}\right)_{\sharp}\left(\overline{\eta^{2}}\right)=i_{1} \circ \pi_{2} \circ \overline{\eta^{2}}=i_{1} \circ \eta^{2} \neq 0 .
$$


Let $f_{12}=s \pi_{2}^{*}\left(i_{1}\right)$. If $s=2 l$ for $1 \leq l \leq d / 2$, then

$$
s \pi_{2}^{*}\left(i_{1}\right)_{\sharp}\left(\overline{\eta^{2}}\right)=2 l i_{1} \circ \eta^{2}=l 2 \eta_{3}=0 .
$$

However, if $s=2 l+1$ for $0 \leq l \leq d / 2-1$, then

$$
s \pi_{2}^{*}\left(i_{1}\right)_{\sharp}\left(\overline{\eta^{2}}\right)=(2 l+1) i_{1} \circ \eta^{2}=\eta_{3} \neq 0 .
$$

Thus, each $f_{12} \in\left\langle 2 \pi_{2}^{*}\left(i_{1}\right)\right\rangle \cong Z_{d / 2}$ induces the trivial homomorphism on $n+3$. However, for $f_{12} \notin\left\langle 2 \pi_{2}^{*}\left(i_{1}\right)\right\rangle$, we have $f_{12 \sharp(n+3)} \neq 0$.

Next, we consider $f_{21 \sharp(n+3)}$. Note that

$$
\left[M_{1}, M_{2}\right] \cong Z_{2} \oplus Z_{2} \oplus Z_{2}\left\{\pi_{1}^{*}\left(\eta_{1}\right), \pi_{1}^{*}\left(\eta_{2}\right), \alpha\right\} .
$$

Since $\alpha_{\sharp(n+2)}=0$, we consider only the generators $\pi_{1}^{*}\left(\eta_{1}\right)$ and $\pi_{1}^{*}\left(\eta_{2}\right)$. For $\pi_{1}^{*}\left(\eta_{1}\right)$, we have

$$
\pi_{1}^{*}\left(\eta_{1}\right)_{\sharp}\left(\eta_{3}\right)=\eta_{1} \circ \pi_{1} \circ \eta_{3}=\eta_{1} \circ \pi_{1} \circ i_{1} \eta^{2}=0
$$

and

$$
\pi_{1}^{*}\left(\eta_{1}\right)_{\sharp}\left(\eta_{4}\right)=\eta_{1} \circ \pi_{1} \circ \eta_{4}=\eta_{1} \circ \eta=i_{2 \sharp}\left(\eta^{2}\right) \circ \eta=4 i_{2 \sharp}(v) .
$$

Thus, if $(p, 24)=4$ or $(p, 24)=12$, then $\pi_{1}^{*}\left(\eta_{1}\right)_{\sharp}\left(\eta_{4}\right)=4 i_{1 \sharp}(v)=0$, and if $(p, 24)=8$ or $(p, 24)=24$, then $\pi_{1}^{*}\left(\eta_{1}\right)_{\sharp}\left(\eta_{4}\right)=4 i_{1 \sharp}(v) \neq 0$.

Since $\pi_{2 \sharp}\left(\eta_{2} \circ \eta\right)=\eta^{2}$, we have $\pi_{1}^{*}\left(\eta_{2}\right)_{\sharp}\left(\eta_{4}\right)=\eta_{2} \circ \pi_{1} \circ \eta_{4}=\eta_{2} \circ \eta \neq 0$.

Therefore, if $(p, 24)=4$ or $(p, 24)=12$, we have

$$
\mathcal{E}_{\sharp}^{\operatorname{dim}+1}(X) \cong\left\{\left(\begin{array}{cc}
1 & f_{12} \\
f_{21} & 1
\end{array}\right) \mid f_{12} \in\left\langle 2 \pi_{2}^{*}\left(i_{1}\right)\right\rangle, f_{21} \in\left\langle\pi_{2}^{*}\left(\eta_{1}\right)\right\rangle\right\},
$$

and if $(p, 24)=8$ or $(p, 24)=24$, we have

$$
\mathcal{E}_{\sharp}^{\operatorname{dim}+1}(X) \cong\left\{\left(\begin{array}{cc}
1 & f_{12} \\
1 & 1
\end{array}\right) \mid f_{12} \in\left\langle 2 \pi_{2}^{*}\left(i_{1}\right)\right\rangle\right\} .
$$

\section{References}

[Araki and Toda 1965] S. Araki and H. Toda, "Multiplicative structures in $\bmod q$ cohomology theories, I", Osaka J. Math. 2 (1965), 71-115. MR 32 \#449 Zbl 0129.15201

[Arkowitz 1990] M. Arkowitz, "The group of self-homotopy equivalences—a survey", pp. 170-203 in Groups of self-equivalences and related topics (Montreal, 1988), edited by R. A. Piccinini, Lecture Notes in Math. 1425, Springer, Berlin, 1990. MR 91i:55001 Zbl 0713.55004

[Arkowitz and Maruyama 1998] M. Arkowitz and K.-i. Maruyama, "Self-homotopy equivalences which induce the identity on homology, cohomology or homotopy groups", Topology Appl. 87:2 (1998), 133-154. MR 99i:55009 Zbl 0929.55008

[Blakers and Massey 1952] A. L. Blakers and W. S. Massey, "The homotopy groups of a triad, II", Ann. of Math. (2) 55 (1952), 192-201. MR 13,485f Zbl 0046.40604

[Gray 1975] B. Gray, Homotopy theory: An introduction to algebraic topology, Pure and Applied Mathematics 64, Academic Press, New York, 1975. MR 53 \#6528 Zbl 0322.55001 
[Hilton 1965] P. Hilton, Homotopy theory and duality, Gordon and Breach, New York, 1965. MR 33 \#6624 Zbl 0155.50801

[Jeong 2010] M. H. Jeong, "Certain subgroups of self-homotopy equivalences of the wedge of two Moore spaces”, Commun. Korean Math. Soc. 25:1 (2010), 111-117. MR 2011a:55008 Zbl 1213.55005

[Puppe 1958] D. Puppe, "Homotopiemengen und ihre induzierten Abbildungen, I", Math. Z. 69 (1958), 299-344. MR 20 \#6698 Zbl 0092.39803

[Spanier 1966] E. H. Spanier, Algebraic topology, McGraw-Hill, New York, 1966. MR 35 \#1007 Zbl 0145.43303

[Toda 1962] H. Toda, Composition methods in homotopy groups of spheres, Annals of Mathematics Studies 49, Princeton University Press, 1962. MR 26 \#777 Zbl 0101.40703

Received August 2, 2013. Revised October 17, 2013.

Ho WON CHOI

DEPARTMENT OF MATHEMATICS

KOREA UNIVERSITY

SEOUL 702-701

SOUTH KOREA

howon@korea.ac.kr

Kee Young LeE

DEPARTMENT OF MATHEMATICS

KOREA UNIVERSITY

SEJONG 425-791

SOUTH KOREA

keyolee@korea.ac.kr 


\title{
PACIFIC JOURNAL OF MATHEMATICS
}

\author{
msp.org/pjm
}

Founded in 1951 by E. F. Beckenbach (1906-1982) and F. Wolf (1904-1989)

\section{EDITORS}

Don Blasius (Managing Editor)

Department of Mathematics

University of California

Los Angeles, CA 90095-1555

blasius@math.ucla.edu

\author{
Paul Balmer \\ Department of Mathematics \\ University of California \\ Los Angeles, CA 90095-1555 \\ balmer@math.ucla.edu \\ Robert Finn \\ Department of Mathematics \\ Stanford University \\ Stanford, CA 94305-2125 \\ finn@math.stanford.edu \\ Sorin Popa \\ Department of Mathematics \\ University of California \\ Los Angeles, CA 90095-1555 \\ popa@math.ucla.edu
}

\author{
Vyjayanthi Chari \\ Department of Mathematics \\ University of California \\ Riverside, CA 92521-0135 \\ chari@math.ucr.edu \\ Kefeng Liu \\ Department of Mathematics \\ University of California \\ Los Angeles, CA 90095-1555 \\ liu@math.ucla.edu \\ Jie Qing \\ Department of Mathematics \\ University of California \\ Santa Cruz, CA 95064 \\ qing@ cats.ucsc.edu
}

\section{PRODUCTION}

Silvio Levy, Scientific Editor, production@msp.org

\section{SUPPORTING INSTITUTIONS}

ACADEMIA SINICA, TAIPEI

CALIFORNIA INST. OF TECHNOLOGY

INST. DE MATEMÁTICA PURA E APLICADA

KEIO UNIVERSITY

MATH. SCIENCES RESEARCH INSTITUTE

NEW MEXICO STATE UNIV.

OREGON STATE UNIV.

\author{
STANFORD UNIVERSITY \\ UNIV. OF BRITISH COLUMBIA \\ UNIV. OF CALIFORNIA, BERKELEY \\ UNIV. OF CALIFORNIA, DAVIS \\ UNIV. OF CALIFORNIA, LOS ANGELES \\ UNIV. OF CALIFORNIA, RIVERSIDE \\ UNIV. OF CALIFORNIA, SAN DIEGO \\ UNIV. OF CALIF., SANTA BARBARA
}

\author{
Daryl Cooper \\ Department of Mathematics \\ University of California \\ Santa Barbara, CA 93106-3080 \\ cooper@math.ucsb.edu \\ Jiang-Hua Lu \\ Department of Mathematics \\ The University of Hong Kong \\ Pokfulam Rd., Hong Kong \\ jhlu@maths.hku.hk \\ Paul Yang \\ Department of Mathematics \\ Princeton University \\ Princeton NJ 08544-1000 \\ yang@math.princeton.edu
}

These supporting institutions contribute to the cost of publication of this Journal, but they are not owners or publishers and have no responsibility for its contents or policies.

See inside back cover or msp.org/pjm for submission instructions.

The subscription price for 2014 is US $\$ 410 /$ year for the electronic version, and \$535/year for print and electronic.

Subscriptions, requests for back issues and changes of subscribers address should be sent to Pacific Journal of Mathematics, P.O. Box 4163, Berkeley, CA 94704-0163, U.S.A. The Pacific Journal of Mathematics is indexed by Mathematical Reviews, Zentralblatt MATH, PASCAL CNRS Index, Referativnyi Zhurnal, Current Mathematical Publications and Web of Knowledge (Science Citation Index).

The Pacific Journal of Mathematics (ISSN 0030-8730) at the University of California, c/o Department of Mathematics, 798 Evans Hall \#3840, Berkeley, CA 94720-3840, is published twelve times a year. Periodical rate postage paid at Berkeley, CA 94704, and additional mailing offices. POSTMASTER: send address changes to Pacific Journal of Mathematics, P.O. Box 4163, Berkeley, CA 94704-0163.

PJM peer review and production are managed by EditFLOW ${ }^{\circledR}$ from Mathematical Sciences Publishers.

\section{PUBLISHED BY}

\section{mathematical sciences publishers \\ nonprofit scientific publishing}

http://msp.org/

(C) 2014 Mathematical Sciences Publishers 


\section{PACIFIC JOURNAL OF MATHEMATICS}

Volume $272 \quad$ No. $1 \quad$ November 2014

Nonconcordant links with homology cobordant zero-framed surgery $\quad 1$ manifolds

JAE CHOON CHA and MARK POWELL

Certain self-homotopy equivalences on wedge products of Moore spaces

Ho Won CHOI and KeE Young LeE

Modular transformations involving the Mordell integral in Ramanujan's lost notebook

Youn-SEO CHOI

The $D$-topology for diffeological spaces

J. DANiEl Christensen, Gordon SinNAMON and ENXIN Wu

On the Atkin polynomials

AHMAD EL-Guindy and Mourad E. H. ISMAIL

Evolving convex curves to constant-width ones by a perimeter-preserving flow

LAIYUAN GaO and Shengliang PAN

Hilbert series of certain jet schemes of determinantal varieties

SudhiR R. GHORPADE, Boyan Jonov and B. A. SeTHuraman

On a Liu-Yau type inequality for surfaces

Oussama Hijazi, Sebastián Montiel and Simon Raulot

Nonlinear Euler sums

ISTVÁN MEZŐ

Boundary limits for fractional Poisson $a$-extensions of $L^{p}$ boundary functions 227 in a cone

LEI QIAO and TAO ZHAO

Jacobi-Trudi determinants and characters of minimal affinizations

STEVEN V SAM

Normal families of holomorphic mappings into complex projective space concerning shared hyperplanes

LiU Yang, Caiyun Fang and Xuecheng Pang 